\title{
Design of Microwave Multibandpass Filters with Quasilumped Resonators
}

\author{
Dejan Miljanović, ${ }^{1,2}$ Milka Potrebić, ${ }^{2}$ and Dejan V. Tošićc \\ ${ }^{1}$ m:tel Bosnia and Herzegovina, Kneza Lazara 4, 74000 Doboj, Bosnia and Herzegovina \\ ${ }^{2}$ School of Electrical Engineering, University of Belgrade, Bulevar Kralja Aleksandra 73, P.O. Box 35-54, 11120 Belgrade, Serbia \\ Correspondence should be addressed to Milka Potrebić; milka_potrebic@etf.rs
}

Received 2 September 2014; Accepted 18 November 2014

Academic Editor: Masoud Hajarian

Copyright (C) 2015 Dejan Miljanović et al. This is an open access article distributed under the Creative Commons Attribution License, which permits unrestricted use, distribution, and reproduction in any medium, provided the original work is properly cited.

\begin{abstract}
Design of RF and microwave filters has always been the challenging engineering field. Modern filter design techniques involve the use of the three-dimensional electromagnetic (3D EM) solvers for predicting filter behavior, yielding the most accurate filter characteristics. However, the 3D EM simulations are time consuming. In this paper, we propose electric-circuit models, instead of 3D EM models, suitable for design of RF and microwave filters with quasilumped coupled resonators. Using the diakoptic approach, the 3D filter structure is decomposed into domains that are modeled by electric networks. The coupling between these domains is modeled by capacitors and coupled inductors. Furthermore, we relate the circuit-element values to the physical dimensions of the 3D filter structure. We propose the filter design procedure that is based on the circuit models and fast circuit-level simulations, yielding the element values from which the physical dimensions can be obtained. The obtained dimensions should be slightly refined for achieving the desired filter characteristics. The mathematical problems encountered in the procedure are solved by numerical and symbolic computations. The procedure is exemplified by designing a triple-bandpass filter and validated by measurements on the fabricated filter. The simulation and experimental results are in good agreement.
\end{abstract}

\section{Introduction}

Traditional approach in filter design has several distinct stages: specification, approximation, synthesis, implementation, and study of imperfections. Main filter characteristics, such as filter type, central frequency, bandwidth, insertion loss, and return loss, are given in the specification stage. Mathematical expressions of physically realizable frequency characteristics are formulated in the approximation stage. Development of an electric circuit with ideal elements, from the approximation stage, is carried out in the synthesis stage. The electric circuit is transformed into the physical device in the implementation stage for a desired technology. Study of imperfections is the final filter design stage when the physical structure is tuned or optimized to compensate for various parasitic effects $[1,2]$.

Particular implementation of the filter elements depends on the chosen technology. If the planar technology is used there are various approaches for filter implementation. These planar filters have been implemented as microstrip filters with open-loop resonators [3], microstrip interdigital filters [4], microstrip SIR (stepped impedance resonator) filters [5], dual-layer filters [6], dual-metal-plane patch-via-spiral filters [7], multilayer filters with apertures on the ground plane [8], and multilayer stacked LC resonator filters [9]. For the above mentioned filter design examples, a common approach is to find relations between geometrical characteristics of the element implementations and the corresponding element values, such as inductances, capacitances, resistances, coupling coefficients, and quality factors ( $Q$-factor). These relations are typically generated by extensive 3D EM simulations but can be obtained experimentally as well. Practically, these relations are presented graphically as a set of curves describing, for example, quality factor as a function of coupling gaps [5], coupling as a function of interresonator spacing and quality factor as a function of tapped-line feed position [9], resonant frequency as a function of resonator dimensions [7], and electric, magnetic, and mixed coupling as a function of apertures 
in the ground plane [10]. Using these data, initial dimensions and mutual positions of element implementations are chosen.

The initial implementation guess, obtained from the set of curves, is refined iteratively in order to meet the filter specifications. The analysis of the actual filter structure and its parts is performed by 3D EM simulators, such as IE3D [11], yielding the most accurate filter characteristics. However, the 3D EM simulations are time consuming and require extensive computer resources, such as CPU speed and the operating memory size. Moreover, the filter parameter refinement often requires repeated 3D EM simulations which further extend the simulation time.

In this paper, we focus on electric-circuit models, instead of 3D EM models, suitable for design of RF and microwave multibandpass filters with quasilumped coupled resonators. The circuit models are composed of linear time-invariant passive lumped elements: capacitors, inductors, coupled inductors, and resistors. Using the diakoptic approach, the $3 \mathrm{D}$ filter structure is decomposed into domains that are modeled by linear time-invariant passive electric networks with finite number of elements. Electromagnetic coupling between the domains is modeled by capacitors (for electrical coupling) and coupled inductors (for magnetic coupling). Compared to the 3D EM simulators, the circuit-level simulators analyze the filter almost instantaneously. Therefore, repeated circuit simulations can be efficiently used to identify the key filter parameters which predominantly affect the filter characteristics. Consequently, these key parameters can be effectively used to fine-tune the filter performance and meet the specifications $[12,13]$.

In recent papers, electric-circuit models for filter design have been often used, as in $[5,7,14]$. They have been used to model filters and to analyze the influence of various filter elements on the filter characteristics. Prior to deploying a circuit model, 3D models of the filter have already been made and elements of the circuit model have been extracted from that $3 \mathrm{D}$ model. Circuit model is actually based on the $3 \mathrm{D}$ model that is to be implemented.

In this paper, the concept of circuit model is used as a primary tool to analyze and design a filter. A structure of the quasilumped coupled-resonator bandpass filter is proposed, for which the electric-circuit model is derived. The filter structure is decomposed and every filter $3 \mathrm{D}$ component is modeled by lumped electric elements. These elements are analytically described and relations between the electricelement values and the geometrical dimensions of the filter structure are established in software [15]. Using circuit models, three single bandpass filters are designed. After fine tuning, these filters are analyzed by 3D EM solvers. Finally, a triple-bandpass filter is designed using the three single bandpass filters. The filter is simulated using the 3D EM model and validated by measurements on the fabricated filter. The simulated results and experimental results are in good agreement.

\section{Electric-Circuit Model of Bandpass Filter}

In this section, we propose a novel microwave bandpass filter design based on coupled resonators. The resonators are

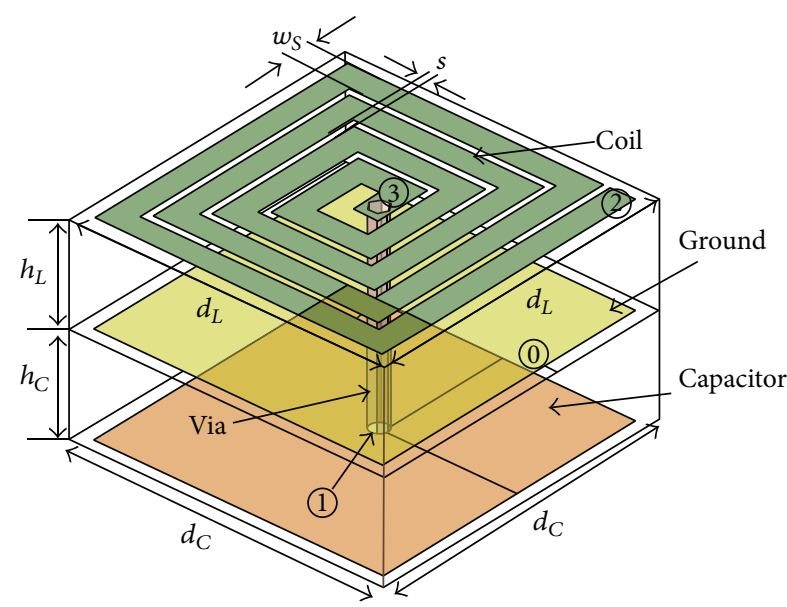

FIGURE 1: 3D EM model of the multilayer quasilumped resonator.

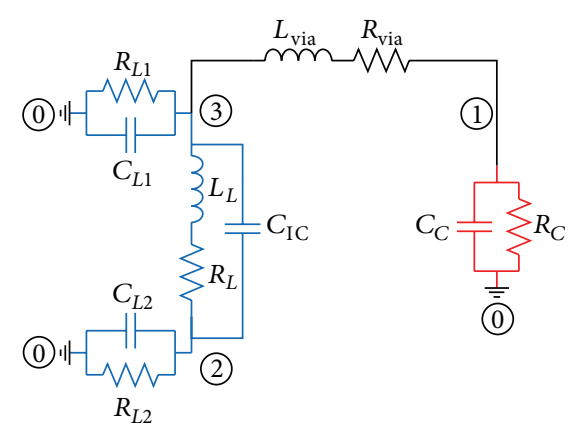

FIGURE 2: Electric-circuit model of the multilayer quasilumped resonator.

implemented with quasilumped elements, using the planar multilayer technology. This approach has been inspired by filter design techniques reported in $[7,9,16]$.

We have made a comprehensive analysis of one type of the multilayer quasilumped resonator and the corresponding feeders $[17,18]$. From the design viewpoint, the filter implementation using these multilayer resonators is very flexible, but the 3D EM simulation is time consuming. In this work, one of our objectives is to reduce the simulation time as much as possible.

The multilayer quasilumped resonator is shown in Figure 1. The resonator is realized using double-sided microstrip. The mid layer is the common ground plane.

The resonator contains three elements: a spiral inductor, a via-hole, and a parallel-plate capacitor. The via connects the inner end of the spiral inductor at the top plane and the capacitor at the bottom plane. The outer end of the spiral inductor is open line. The via passes through the structure without electrical connection to the common ground plane.

The points 1 to 3 are marked on the resonator structure (Figure 1) in order to decompose the resonator into domains that are modeled by electrical networks. The points of the filter structure correspond to electric-circuit nodes. An electric-circuit model of the resonator is shown in Figure 2. 


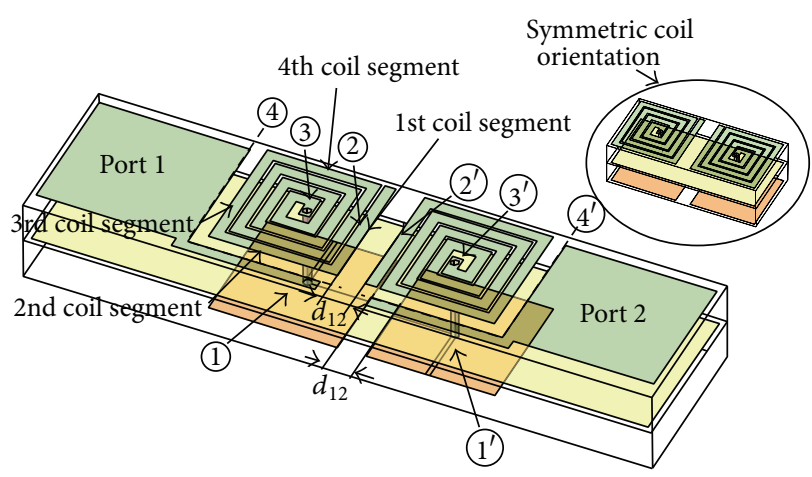

(a)

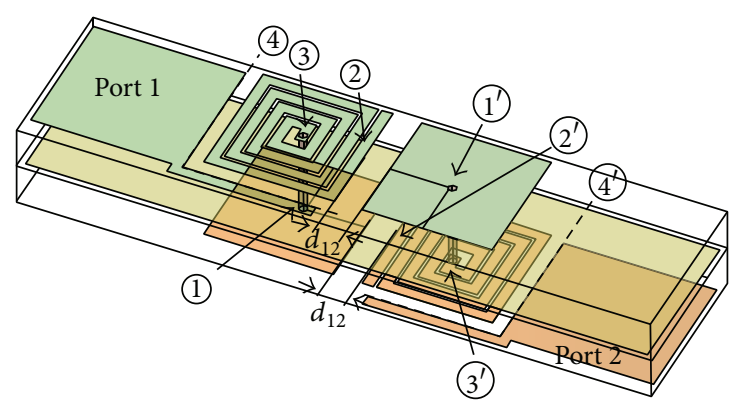

(b)

FIGURE 3: 3D EM model of the second-order bandpass filter with (a) LLCC interresonator coupling, (b) LCLC interresonator coupling.

In this circuit model, the square-spiral inductor is modeled with $\pi$ electric network between nodes 2 and 3 , and the element parameters are inductance $\left(L_{L}\right)$, resistance $\left(R_{L}\right)$, and parasitic capacitances $\left(C_{L 1}\right.$ and $\left.C_{L 2}\right)$ with their resistances $\left(R_{L 1}\right.$ and $\left.R_{L 2}\right)$. The interspiral capacitance $\left(C_{\mathrm{IC}}\right)$ is capacitance between the turns of the spiral inductor.

The parameters of the cylindrical via-hole are inductance $\left(L_{\text {via }}\right)$ and resistance $\left(R_{\text {via }}\right)$ between nodes 1 and 3.

The parameters of the capacitor are capacitance $\left(C_{C}\right)$ and resistance $\left(R_{C}\right)$ between nodes 1 and 0 .

Neglecting the via inductance and all circuit losses modeled by resistors, the parallel resonant frequency of the unloaded resonator (Figure 2) is

$$
=\frac{1}{2 \pi \sqrt{L_{L}\left(\left(C_{C}+C_{L 1}\right) C_{L 2} /\left(\left(C_{C}+C_{L 1}\right)+C_{L 2}\right)+C_{\mathrm{IC}}\right)}},
$$

which is determined by the inductance $L_{L}$ and capacitances $C_{\mathrm{IC}}, C_{C}, C_{L 1}$, and $C_{L 2}$.

This resonator is used as a main building block for the bandpass filter design. In this research, the secondorder bandpass filter is designed, using coupled resonators, as shown in Figure 3. The nodes are marked on the filter resonators ( 1 to 4 for the first and $1^{\prime}$ to $4^{\prime}$ for the second resonator), as shown in Figure 3.

There are two possibilities of the interresonator coupling. In the first case, we have inductor-to-inductor coupling on one side of substrate and capacitor-to-capacitor coupling on the other side. This type of coupling is shown in Figure 3(a) and it will be denoted as $L L C C$ coupling (meaning inductor $L$ to inductor $L$ and capacitor $C$ to capacitor $C$ ). In the second case, we have inductor-to-capacitor coupling on both sides of the layers. This type of coupling is shown in Figure 3(b) and will be denoted as LCLC coupling. These two possibilities require different models of the interresonator coupling. Two electric circuits are shown in Figure 4, corresponding to these two cases of interresonator coupling.

The LLCC coupling is modeled by capacitor (with capacitance $C_{C C}$ ) between two patch capacitors (nodes 1 and $1^{\prime}$ ).
The capacitance between the end of two inductors (nodes 2 and $2^{\prime}$ ) is modeled by capacitor $C_{L L}$. The mutual inductance $\left(M_{\text {total }}=k_{m} L_{L}\right)$ is modeled with inductive coupling between the inductors. The sign of mutual inductance $\left(M_{\text {total }}\right)$ can be positive or negative, depending on the mutual inductor orientation. Symmetric orientation of the inductors is also depicted in Figure 3(a). If we adopt the dot marking convention as in Figure 4(a), then $M_{\text {total }}$ is positive for the symmetric inductor orientation and negative for the asymmetric inductor orientation.

For the LCLC interresonator coupling, there is dominantly capacitive coupling between inductor and capacitor, and it is modeled by capacitance $C_{L C}$ (between nodes 1 and $2^{\prime}, 1^{\prime}$ and 2), as in Figure 4(b).

The feeder is realized as a microstrip line whose width corresponds to the characteristic impedance of $50 \Omega\left(Z_{F}=\right.$ $50 \Omega$ ). The feeder is capacitively coupled to the resonator and it is dominantly coupled to the outer end of the resonator inductor. This is modeled by capacitance $C_{F}$ between nodes 2 and $4\left(2^{\prime}\right.$ and $\left.4^{\prime}\right)$.

\section{Analytic Closed-Form Expressions for Quasilumped Element Values}

In order to analyze and design filter characteristics, using the electric-circuit model, it is necessary to make a correlation between the circuit-element values and the physical dimensions of the 3D filter structure. For that purpose, we use closed-form expressions for inductance, capacitance, and resistance and a software package for finding matrix parameters of the transmission lines [15]. In this research, the substrate used for filter design is RT/Duroid $5880\left(\varepsilon_{r}=2.2\right.$, $\tan \delta=0.001$, substrate thickness $h_{L}=h_{C}=1.575 \mathrm{~mm}$, metallization thickness $t=18 \mu \mathrm{m}$ ).

The capacitance of a rectangular parallel-plate structure must be calculated considering the effect of fringing field. This is important when the size of the gap separating the electrodes (i.e., substrate thickness) is comparable to the dimensions of the capacitor plates. In that case, the capacitance cannot be calculated as for a simple model of 


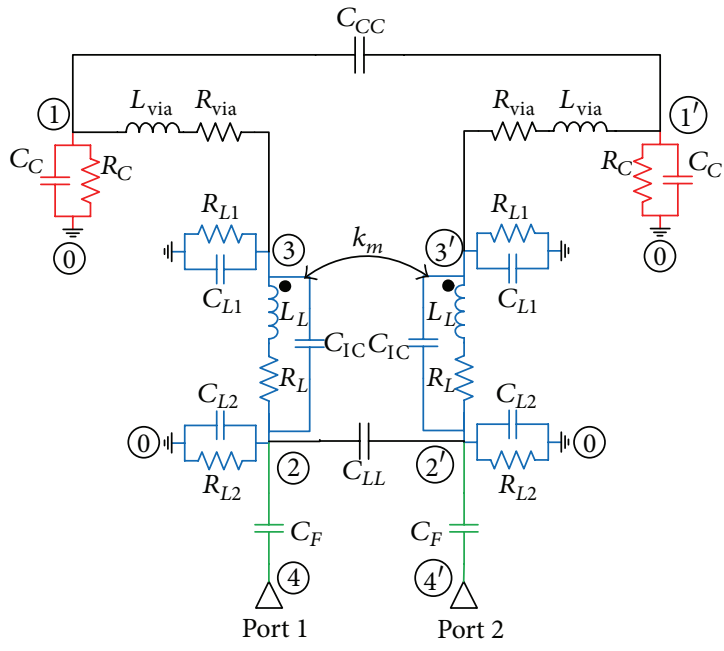

(a)

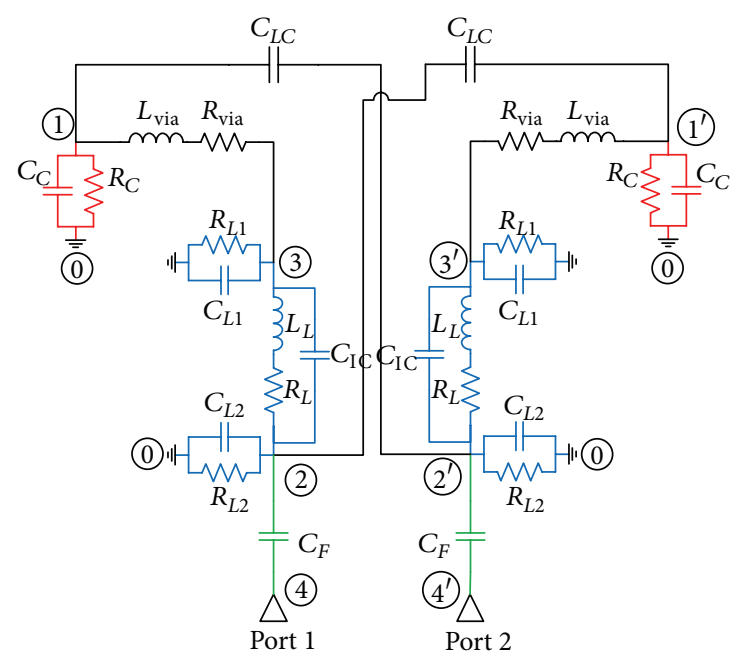

(b)

FIGURE 4: Electric-circuit models of the second-order bandpass filters shown in (a) Figure 3(a) and (b) Figure 3(b).

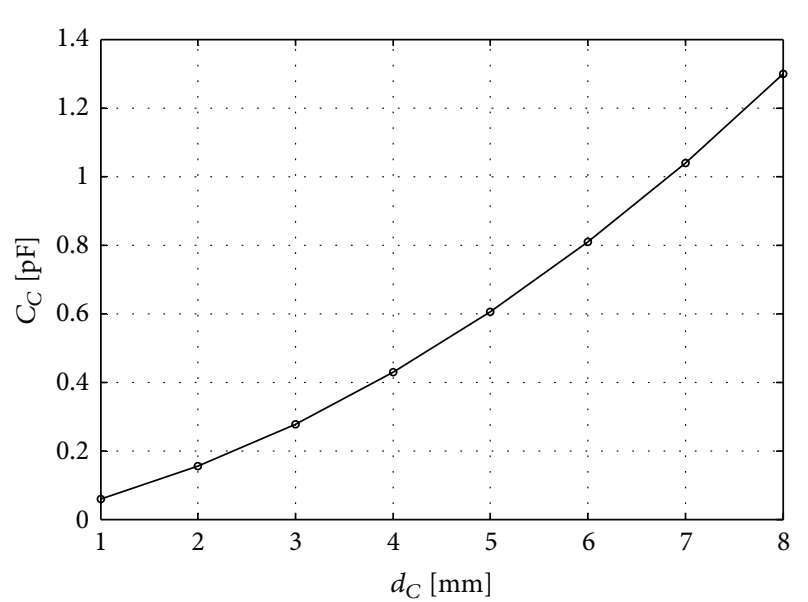

FIGURE 5: Capacitance of the patch capacitor with respect to the outer capacitor dimension $d_{C}$.

parallel-plate devices. The effective capacitance is greater in this case and should be calculated as [19]

$$
\begin{aligned}
C= & \varepsilon_{0} \varepsilon_{r} \frac{d_{X} d_{Y}}{d_{\text {sub }}}\left(1+\frac{d_{\text {sub }}}{\pi d_{X}}\left(1+\ln \left(\frac{2 \pi d_{X}}{d_{\text {sub }}}\right)\right)\right) \\
& \times\left(1+\frac{d_{\text {sub }}}{\pi d_{Y}}\left(1+\ln \left(\frac{2 \pi d_{Y}}{d_{\text {sub }}}\right)\right)\right),
\end{aligned}
$$

where $d_{X}$ and $d_{Y}$ are outer capacitor dimensions (in our case $d_{X}=d_{Y}=d_{C}$ ) and substrate thickness $h_{C}=d_{\text {sub. }}$. The capacitance of the patch capacitor with respect to the capacitor size is show in Figure 5.

The inductance of cylindrical via hole in microstrip can be calculated as a modified inductance of a cylindrical conductor and it will be calculated using the following formula [20]:

$$
\begin{aligned}
L_{\mathrm{via}}=\frac{\mu_{0}}{2 \pi}[ & l_{\mathrm{via}} \ln \left(\frac{l_{\mathrm{via}}+\sqrt{r_{\mathrm{via}}^{2}+l_{\mathrm{via}}^{2}}}{r_{\mathrm{via}}}\right) \\
& \left.+\frac{3}{2}\left(r_{\mathrm{via}}-\sqrt{r_{\mathrm{via}}^{2}+l_{\mathrm{via}}^{2}}\right)\right] .
\end{aligned}
$$

For the chosen substrate and intended via radius $\left(r_{\text {via }}\right)$, the obtained value is $L_{\text {via }}=1.454 \mathrm{nH}$ (length $l_{\text {via }}=h_{L}+h_{C}=$ $3.15 \mathrm{~mm}$ and radius $\left.r_{\text {via }}=0.15 \mathrm{~mm}\right)$. The via resistance is neglected.

The relation between the square-spiral inductance and the square-spiral physical dimensions is given by the following formula [12]:

$$
\begin{aligned}
L= & \frac{1.27 \mu_{0} N^{2}}{2}\left(\frac{d_{L}+d_{i}}{2}\right) \\
& \times\left(\ln \left(\frac{2.07}{\rho}\right)+0.18 \rho+0.13 \rho^{2}\right) K_{g} .
\end{aligned}
$$

The parameter $N$ denotes the number of inductor turns, $d_{L}$ is the outside diameter and $d_{i}$ is the inside diameter of the inductor, $\rho=\left(d_{L}-d_{\mathrm{i}}\right) /\left(d_{L}+d_{i}\right)$ is the fill ratio, and $K_{g}$ is a correction factor for the presence of a ground plane. $K_{g}$ is calculated as follows:

$$
K_{g}=0.57-0.145 \ln \left(\frac{w_{S}}{h_{L}}\right), \quad \frac{w_{S}}{h_{L}}>0.05 .
$$

Using formula (4) one can find the spiral line width $\left(w_{S}\right)$, the turn spacing $(s)$, and the outside diameter $\left(d_{L}\right)$ for the required square-spiral inductance. The approximate maximum number of the inductor turns (for given $d_{L}$ ) is 


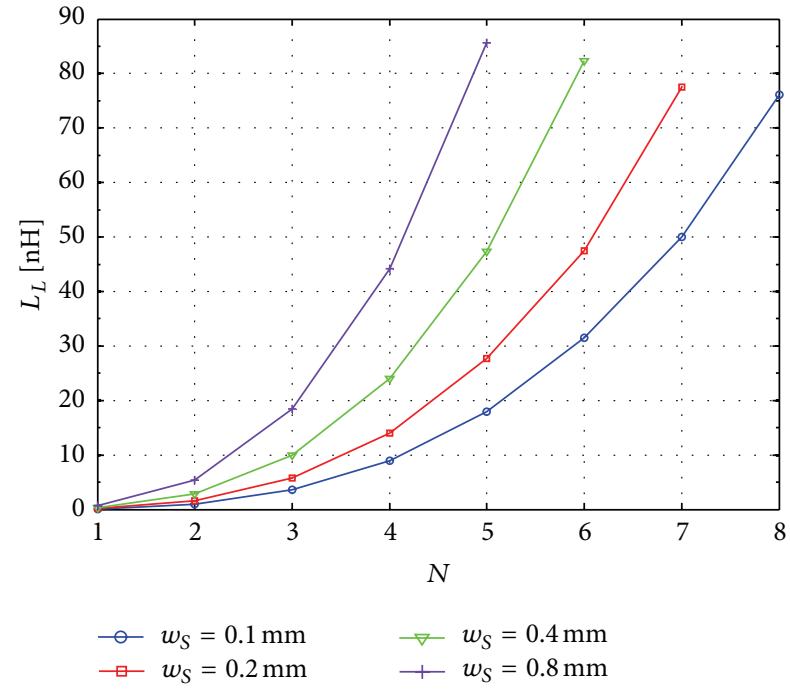

Figure 6: Inductance of the spiral inductor with respect to the number of turns $(N)$ for the turn spacing $s=0.1 \mathrm{~mm}$ and the spiral line widths $w_{S}=0.1,0.2,0.4,0.8 \mathrm{~mm}$.

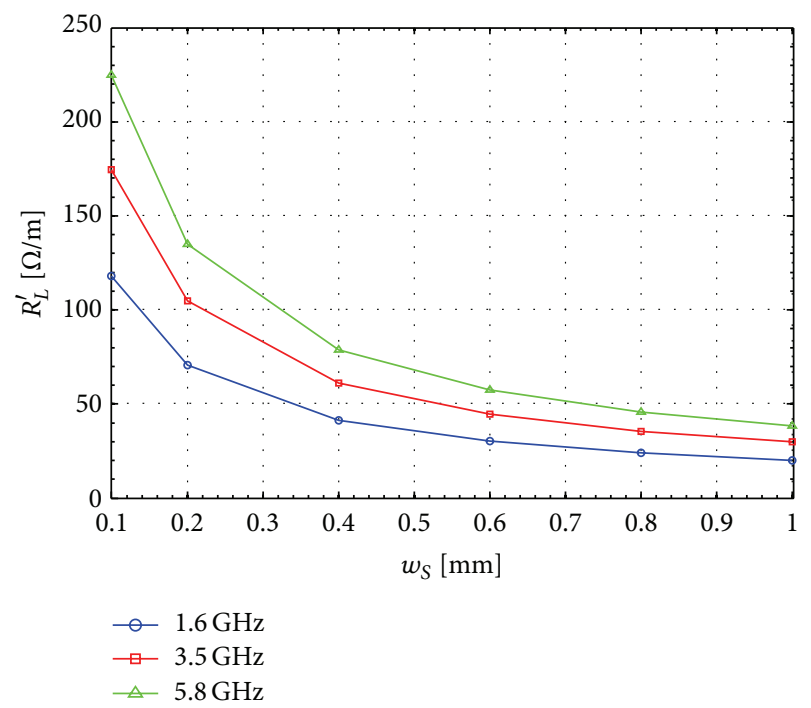

FIgURE 7: Per unit length resistance of the spiral inductor with respect to the spiral line width $w_{S}$ at frequencies $1.6 \mathrm{GHz}, 3.5 \mathrm{GHz}$, and $5.8 \mathrm{GHz}$.

$N=d_{L} /\left(2\left(s+w_{\mathrm{S}}\right)\right)$, in which case $\rho=1$. Using this formula and setting $\rho=1$ in (4), we can derive formula for the maximum theoretically achievable inductance for given $d_{L}$, $s$, and $w_{\mathrm{S}}$ :

$$
L[\mathrm{nH}]=\frac{103.5 d_{L}^{3}}{\left(s+w_{\mathrm{S}}\right)^{2}} .
$$

This formula can be useful for the first estimation of the inductor size. The square-spiral inductance with respect to the number of turns is shown in Figure 6, without considering physical limitations during fabrication.
The parasitic capacitance of the inductor toward ground plane may be calculated using (2) with $d_{X}=d_{Y}=d_{L}$. Although layout of the inductor is different from the layout of the capacitor, our research has shown that (2) can be used with great accuracy in the case of greater inductor fill ratio. This is certainly correct in the case of our filter design objectives, where one of the goals is miniaturization. In our model, the parasitic capacitance of the inductor toward ground plane is equally distributed to $C_{L 1}$ and $C_{L 2}$; that is, $C_{L 1}=C_{L 2}=C_{C} / 2$.

The interspiral capacitance causes parallel resonances of the spiral inductor and it has influence on effective inductance at higher frequencies. The satisfactory estimation of the capacitance value is obtained with [21]

$$
C_{\mathrm{IC}}=\frac{\varepsilon_{\mathrm{reff}} l_{\mathrm{S}} t}{s}
$$

where $\varepsilon_{\text {reff }}$ is the effective relative permeability for the spiral line of width $w_{S}$ and the given substrate. The parameter $l_{S}$ is the average spiral length.

The inductor resistance $\left(R_{L}\right)$ is calculated by multiplying the spiral length with per unit length resistance of microstrip transmission line of width $w_{S}$. Per unit length resistance is frequency dependent, and it is obtained using a software package for calculating matrix parameters of the transmission lines [15]. This dependence is shown in Figure 7.

The mutual inductance between two square-spiral inductors can be calculated using approach presented in [12]. The method is based on formula for the mutual inductance for two adjacent sections of lengths $l_{i}$ and $l_{j}$, of the same width $w_{S}$ and mutual spacing $D_{i j}$ (Figure $8(\mathrm{a})$ ):

$$
\begin{aligned}
& M_{i, j}[\mathrm{nH}] \\
& =2 \cdot 10^{-4} l_{e} \\
& \quad \times\left[\ln \left(\frac{l_{e}}{D_{i j}}+\left(1+\frac{l_{e}^{2}}{D_{i j}^{2}}\right)^{1 / 2}\right)-\left(1+\frac{D_{i j}^{2}}{l_{e}^{2}}\right)^{1 / 2}+\frac{D_{i j}}{l_{e}}\right],
\end{aligned}
$$

where all dimensions are in microns. The parameter $l_{e}$ is the effective length of the two sections between which the mutual inductance is being calculated. As an approximation, $l_{e}$ can be considered as an average length of these two sections, $l_{e}=$ $\left(l_{i}+l_{j}\right) / 2$.

The method for calculating the total mutual inductance is shown in Figure 8(a). The total mutual inductance is calculated as a sum of mutual inductances of each pair of parallel sections, positioned opposite to each other. There is mutual inductance between each segment of the left spiral inductor (denoted from $i=1$ to $2 N$ ) and each opposite segment of the right spiral inductor (denoted from $j=1$ to $2 N$ ). In this case, we assume that $N$ is the number of complete turns. Mutual inductances of nonopposite segments are neglected. For the adopted current direction, positive and 


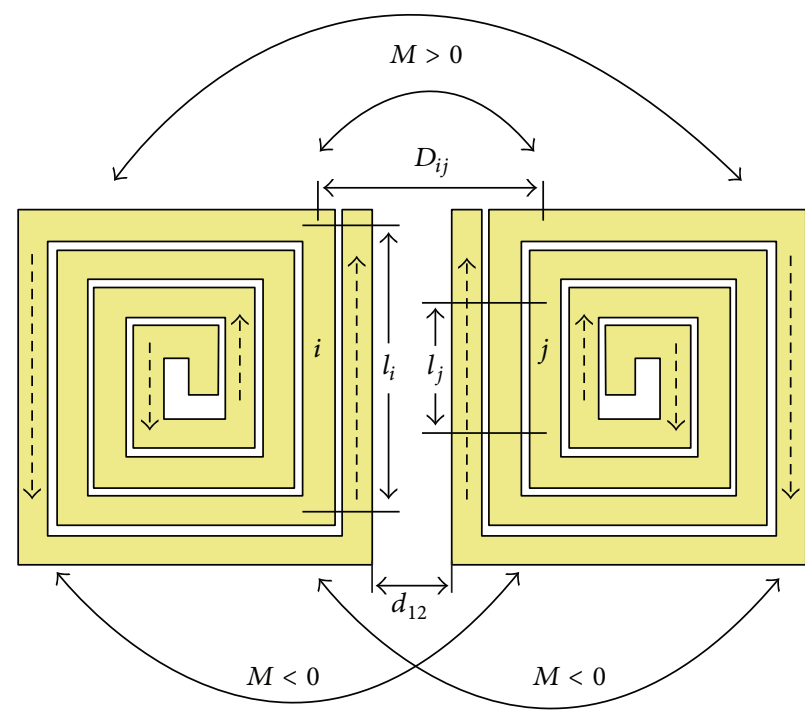

(a)

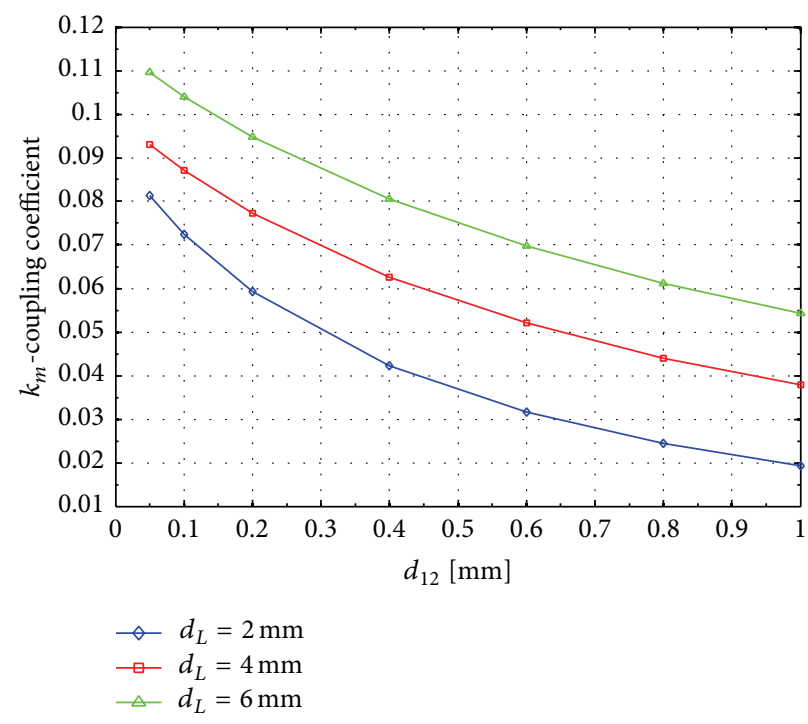

(b)

FIGURE 8: (a) Coupled spiral inductors and (b) coupling coefficient $\left(k_{m}\right)$ with respect to interinductor spacing $\left(d_{12}\right)$ for $N=4$ and the outside diameter of the inductor $d_{L}=2,4,6 \mathrm{~mm}$.

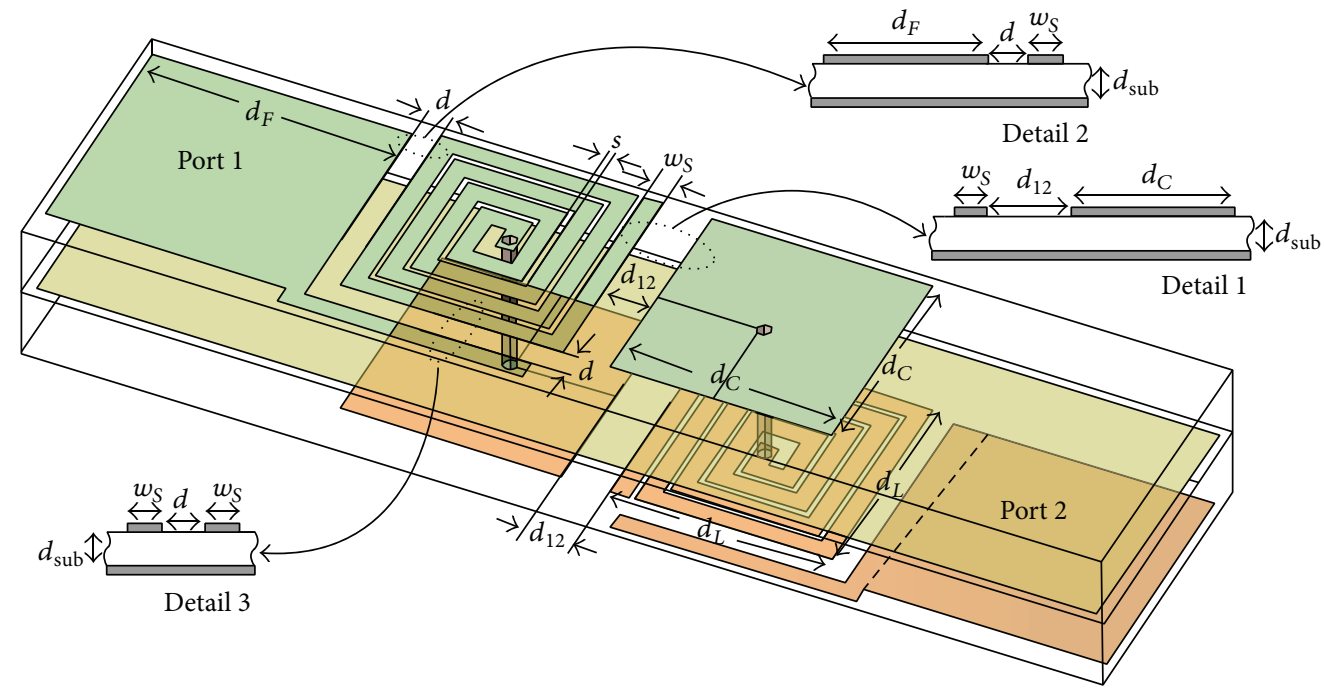

Figure 9: The second-order bandpass filter structure.

negative mutual inductances are marked. The total mutual inductance can be calculated as follows:

$$
\begin{aligned}
M_{\text {total }}= & \sum_{i=1}^{N} \sum_{j=1}^{N} M_{i, j}+\sum_{i=N+1}^{2 N} \sum_{j=N+1}^{2 N} M_{i, j}-\sum_{i=1}^{N} \sum_{j=N+1}^{2 N} M_{i, j} \\
& -\sum_{i=N+1}^{2 N} \sum_{j=1}^{N} M_{i, j}=\sum_{i=1}^{N} \sum_{j=1}^{N} M_{i, j} \\
& +\sum_{i=N+1}^{2 N} \sum_{j=N+1}^{2 N} M_{i, j}-2 \sum_{i=1}^{N} \sum_{j=N+1}^{2 N} M_{i, j}
\end{aligned}
$$

where $M_{i, j}$ is given by (8) and $M_{i, j}=M_{j, i}$ for $i=1$ to $N, j=N+1$ to $2 N$. In the case of the asymmetrical inductor orientation, the total mutual inductance has the opposite algebraic sign. Formula (9) is primarily used to obtain the maximum achievable value of the mutual inductance, because a greater mutual inductance value can cause appearance of transmission zeros. As an illustration, the coupling coefficient $\left(k_{m}=M_{\text {total }} / L_{L}\right)$ is shown in Figure 8(b), with respect to the distance between spiral inductors $\left(d_{12}\right)$, for several inductor sizes $\left(d_{L}\right)$ and $N=4$.

The capacitances between the resonators and capacitance between the feeder and the resonator can be found using a software package for calculating matrix parameters of the coupled transmission lines [15]. This approach can be explained by the details shown in Figures 9 and 3 .

The capacitance $C_{L L}$ is equal to the mutual capacitance (sometimes referred to as the coupling capacitance) of a short 


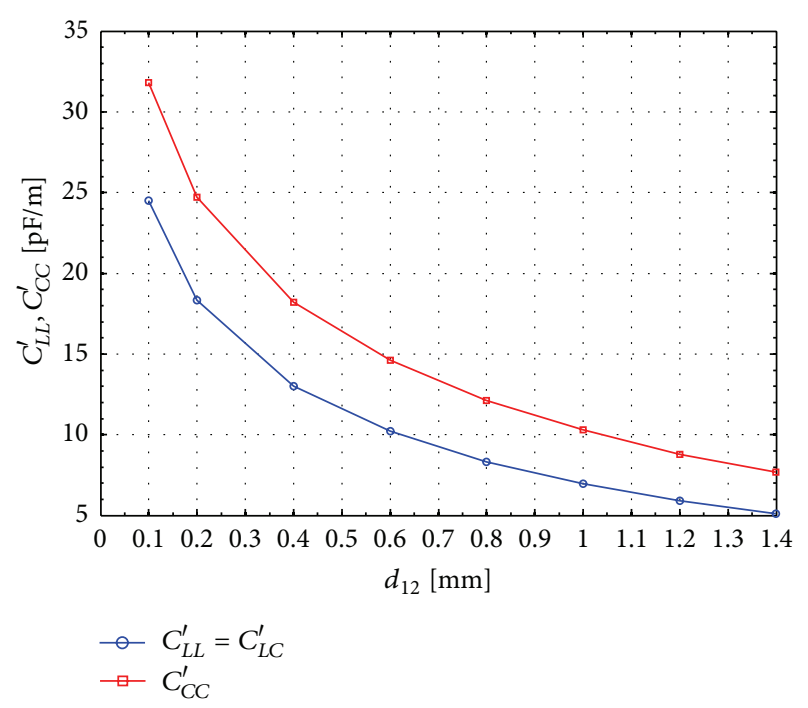

Figure 10: Per unit length capacitance $\left(C_{L L}^{\prime}, C_{C C}^{\prime}\right.$, and $\left.C_{L C}^{\prime}\right)$ with respect to the interresonator distance $\left(d_{12}\right)$.

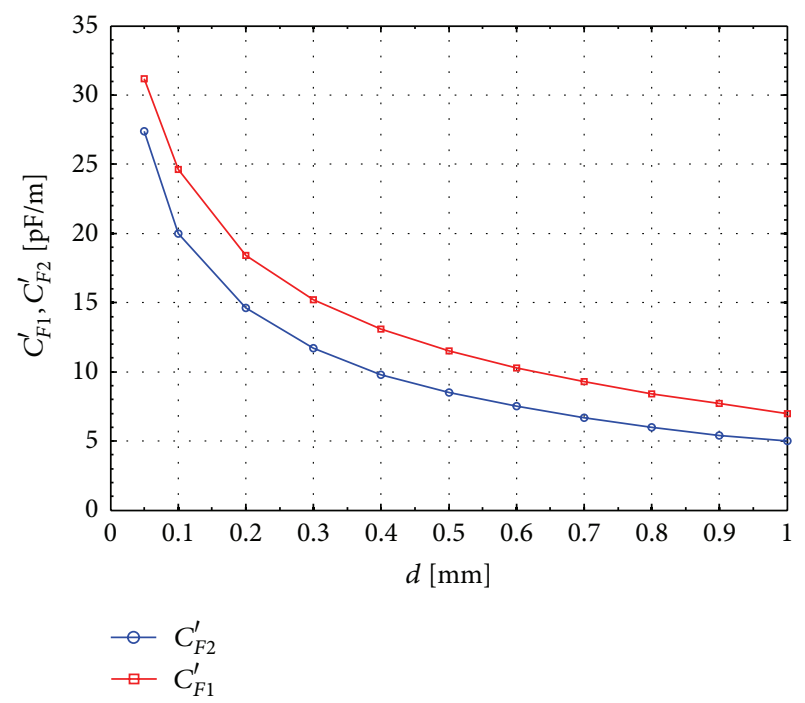

FIgURE 11: Per unit length capacitance $\left(C_{F 1}^{\prime}\right.$ and $\left.C_{F 2}^{\prime}\right)$ with respect to distance $(d)$ between the feeder and the square-spiral inductor.

section (length $d_{L}$ ) of coupled lines of the same width $\left(w_{S}\right)$, mutually separated by the distance $d_{12}$ (Figure 3(a)). The capacitance $C_{C C}$ is equal to the mutual capacitance of a short section (length $d_{C}$ ) of coupled lines of the same width $\left(d_{C}\right)$, mutually separated by the distance $d_{12}$ (Figure 3(a)).

The capacitance $C_{L C}$ is equal to the mutual capacitance of a short section (length $d_{C}=d_{L}$ ) of coupled lines of widths $d_{C}$ and $w_{S}$, mutually separated by the distance $d_{12}$ (Figure 9 , detail 1).

The capacitance $C_{F}$ is the sum of the capacitance between the central part of the feeder and the resonator $\left(C_{F 1}\right)$ and the capacitance between the side part of the feeder and the resonator $\left(C_{F 2}\right)$. The first one is equal to the mutual capacitance of a short section (length $d_{L}$ ) of coupled lines of widths $d_{F}$ and $w_{S}$, mutually separated by the distance $d$ (Figure 9, detail 2). The second one is equal to the mutual capacitance of a short section (length $d_{L}$ ) of coupled lines of the same width $\left(w_{S}\right)$, mutually separated by the distance $d$ (Figure 9, detail 3).

The values of these capacitances are presented in Figures 10 and 11 , for dimensions of interest.

We are able to estimate the values of each element in the circuit model and the corresponding physical dimensions using formulas and graphs presented in this section.

\section{Influence of Circuit-Element Values on Filter Frequency Response}

Using the electric-circuit model, it is now possible to make fast analysis of the influence of every element on the filter frequency response. It has been shown that the variation of the spiral inductor parameters has the most significant influence on the resonant frequency. The resonant frequency is mostly determined by the spiral inductance $L_{L}$ and capacitances $C_{\mathrm{IC}}$, $C_{L 1}$, and $C_{L 2}$. The value of capacitance $C_{C}$ also influences resonant frequency, but it is less significant. The influence of the via becomes more pronounced at higher frequencies. The interspiral capacitance $C_{\text {IC }}$ also has significant influence on filter selectivity. Greater $C_{\mathrm{IC}}$ increases filter selectivity but also decreases resonant frequency. A possible way to increase this value is to use greater number of spiral turns and smaller turn spacing $s$. As expected, $C_{L C}, C_{C C}, C_{L L}$, $k_{m}$, and $C_{F}$ determine filter bandwidth, insertion loss, and return loss. The above observations imply that the main resonator element for adjusting filter characteristics is the spiral inductor. Variation of the inductor parameters changes resonant frequency and selectivity simultaneously.

By analytically solving [22] the circuit model for the resonator (Figure 4(a) with $C_{C C}=C_{L L}=0$ and $k_{m}=0$, or Figure 4(b) with $C_{L C}=0$ ), the following formula for the resonant frequency is obtained:

$$
f_{0}=\sqrt{\frac{(2 \pi)^{-2} L_{L}^{-1}\left(C_{C}+C_{L 1}+C_{L 2}+C_{F}\right)}{\left(C_{C}+C_{L 1}+C_{\mathrm{IC}}\right)\left(C_{L 2}+C_{F}\right)+\left(C_{C}+C_{L 1}\right) C_{\mathrm{IC}}}} .
$$

The most important phenomenon is that filters using two different types of interresonator coupling have different overall characteristics. $L C L C$ interresonator coupling always generates transmission zeros, while LLCC interresonator coupling does not. This effect for $L L C C$ and $L C L C$ interresonator coupling is shown in Figure 12. Using LLCC interresonator coupling, there is a negative electric coupling $k_{e}$ between the capacitors (through $C_{C C}$ ) and positive or negative magnetic coupling $k_{m}$ between the inductors (through $M_{\text {total }}$ ). If these two couplings have the opposite signs and $k_{m}$ has sufficiently high value, transmission zeros can emerge. The opposite signs of $k_{e}$ and $k_{m}$ can be provided only with the symmetric inductor orientation in the resonators. Sufficiently high positive value of $k_{m}$ can be achieved using a different patchvia-spiral approach [7]. However, the resonator realization in [7] has grounded inductors which results in a greater 


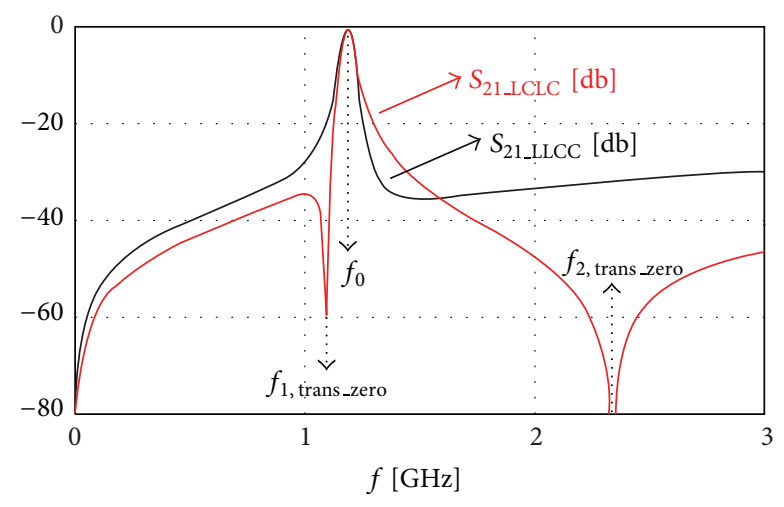

FIGURE 12: Frequency responses of the filters shown in Figure 4 for $L L C C$ and LCLC interresonator coupling.

magnetic coupling $k_{m}$. Using the equivalent circuit proposed here, it can be found that, in the case of LLCC interresonator coupling, the value of $k_{m}>0.15$ would be needed in order to have transmission zeros. Such a great value cannot be implemented using the proposed resonator layout.

In the case of $L C L C$ interresonator coupling, the existence of zeros is caused by another phenomenon-the resonance of the inductor and certain capacitors. The resonator equivalent circuit proposed here has a complex inductor model. Therefore, the frequencies at which the transmission zeros occur can be found using the electric-circuit model and modern mathematical tools for expression simplification [22]. This yields to the following formulas for $L C L C$ interresonator coupling (Figure 4(b)):

$$
\begin{gathered}
f_{1, \text { trans_zero }}=\frac{1}{2 \pi \sqrt{L_{L}\left(C_{C}+C_{L 1}+C_{\mathrm{IC}}+C_{L C}\right)}}, \\
f_{2, \text { trans_zero }}=\frac{1}{2 \pi \sqrt{L_{L} C_{\mathrm{IC}}}} .
\end{gathered}
$$

According to formulas (11) it is evident that the resonance of the spiral inductor and the surrounding capacitors is causing the appearance of the transmission zeros.

\section{Filter Design Using Electric-Circuit Model}

In this section, we present the novel procedure for the bandpass filter design using the electric-circuit model (from Figure 4(b)) and relations between lumped-element values and their physical dimensions (Section 3 ). The procedure steps are as follows.

(1) In the first step, lumped-element values of the electric-circuit model are adjusted to meet the designed central frequency and the bandwidth. The most efficient way is to adopt initial dimensions of the resonator in terms of $d_{L}$ and $d_{C}\left(d_{L}=d_{C}\right)$ and the dimensions of the spiral inductor $w_{S}$ and $s$. In our case, with the goal of miniaturization and available milling technique [23], we will adopt dimensions of the square-spiral inductor $w_{S}=0.15 \mathrm{~mm}$ and $s=0.1 \mathrm{~mm}$. This will give us approximate values of $C_{C}, C_{L 1}, C_{L 2}$, and $C_{\mathrm{IC}}$. The only elements now left for adjusting are $L_{L}$ for the central frequency adjustment; $C_{F}$ for return loss; $C_{L C}, C_{L L}, C_{C C}$, and $k_{m}$ for insertion loss and bandwidth adjustment. We obtain values of $C_{F}, C_{L C}, C_{L L}, k_{m}$, and $C_{C C}$ after adjusting frequency response for the circuit model. Although this overall model gives the value of $L_{L}$, this inductance is notably affected by the influence of feeder capacitance $C_{F}$ and interresonator capacitances. The observed value of $L_{L}$ is actually the effective spiral inductance. Therefore, we propose to estimate the actual value of $L_{L}$ by observing unloaded resonator. The actual value of the inductance will give us actual value of the spiral inductor size. This approach means removing interresonator coupling by setting $C_{L C}=C_{L L}=C_{C C}=0, k_{m}=0$, and reducing the influence of feeder by decreasing value of $C_{F}$, while observing $S_{11}$-parameter of the circuit model. $C_{F}$ should be decreased until it has no influence on resonant frequency (observing the $S_{11^{-}}$ parameter). Now, setting the value of $L_{L}$ to adjust the desired resonator central frequency will give us the actual value of the spiral inductance. Tuned this way, the spiral inductance is found to be the most accurate.

(2) In the second step, feasibility of the obtained lumpedelement values is checked using formulas and design curves. If necessary, the first step is repeated and values are modified in order to find feasible solution. In a few iterations, the first variant should be easily achieved.

(3) The third step is realization of 3D EM filter model, performing full 3D EM simulation and comparing the obtained and specified frequency responses.

(4) In the final step, necessary modifications are performed on the 3D EM model and the final simulation and validation has to be done.

In the rest of the text, $L C L C$ interresonator coupling will be considered only, because it provides realizable transmission zeros.

Using the presented approach, we have designed three bandpass filters, whose frequency responses are shown in Figure 13. These filters are designed for central frequencies of $1.6 \mathrm{GHz}$ (Band-I), $3.5 \mathrm{GHz}$ (Band-II), and $5.8 \mathrm{GHz}$ (BandIII), with fractional bandwidths (FBW) of $4 \%$ (Band-I), $4.5 \%$ (Band-II), and 7.5\% (Band-III). These bands are used for GPS navigation, WiMAX access, and $\mathrm{WiFi}$, respectively.

With adopted resonator dimensions $d_{L}^{I}=d_{C}^{I}=3.2 \mathrm{~mm}$, $d_{L}^{\mathrm{II}}=d_{C}^{\mathrm{II}}=2.4 \mathrm{~mm}$, and $d_{L}^{\mathrm{III}}=d_{\mathrm{C}}^{\mathrm{III}}=2 \mathrm{~mm}$ (superscripts I, II, and III will denote parameters for $1.6 \mathrm{GHz}, 3.5 \mathrm{GHz}$, and $5.8 \mathrm{GHz}$ bands, resp.) corresponding lumped-element values are given in Table 1 . The via inductance and all circuit losses are neglected, except the square-spiral inductor resistance $\left(R_{L}\right)$.

Using the values from Table 1 and formulas for finding physical dimensions of the resonator, the distance between the feeder and the resonator and the interresonator distance 


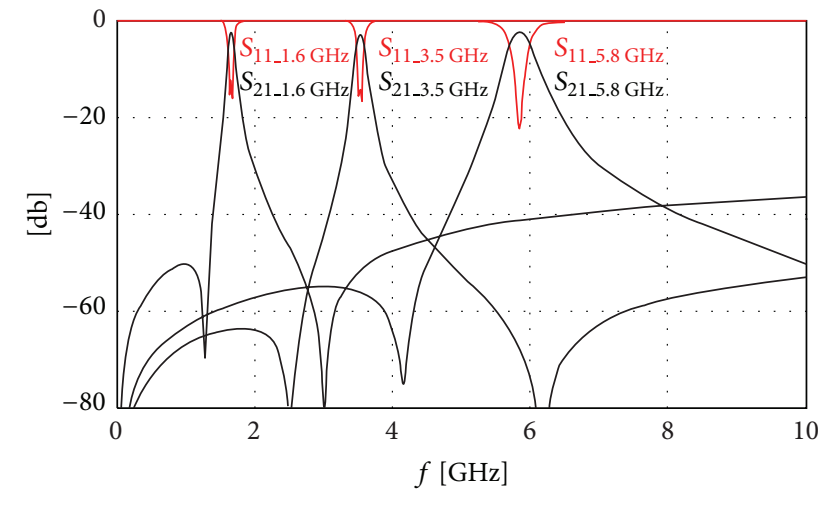

FIGURE 13: Frequency response of three bandpass filters generated by circuit-level simulations.

TABLE 1: Circuit-element values for desired frequency response in Figure 13.

\begin{tabular}{lccc}
\hline$f_{0}[\mathrm{GHz}]$ & $1.6 \mathrm{GHz}$ & $3.5 \mathrm{GHz}$ & $5.8 \mathrm{GHz}$ \\
\hline$C_{C}[\mathrm{pF}]$ & 0.3 & 0.2 & 0.15 \\
\hline$C_{L 1}, C_{L 2}[\mathrm{pF}]$ & 0.15 & 0.1 & 0.075 \\
\hline$R_{L}[\Omega]$ & 3.5 & 2.4 & 1.7 \\
\hline$C_{\mathrm{IC}}[\mathrm{pF}]$ & 0.09 & 0.05 & 0.025 \\
\hline$C_{F}[\mathrm{pF}]$ & 0.3 & 0.1 & 0.1 \\
\hline$C_{L C}[\mathrm{pF}]$ & 0.03 & 0.01 & 0.01 \\
\hline \multirow{2}{*}{$L_{L}[\mathrm{nH}]$} & 30.3 & 11.4 & 5.2 \\
& 49.5 & 16.5 & 8.8 \\
& actual & actual & actual \\
\hline
\end{tabular}

can be found: $N^{\mathrm{I}}=3.8, N^{\mathrm{II}}=2.4, N^{\mathrm{III}}=1.7, d^{\mathrm{I}}=d^{\mathrm{II}}=d^{\mathrm{III}}=$ $0.1 \mathrm{~mm}, d_{12}^{\mathrm{I}}=0.8 \mathrm{~mm}, d_{12}^{\mathrm{II}}=1.5 \mathrm{~mm}$, and $d_{12}^{\mathrm{III}}=1.4 \mathrm{~mm}$. Using these dimensions, three single bandpass filters have been realized and simulated. The obtained results, along with the corresponding filter layouts, are shown in Figure 14.

In the next step, it was necessary to make small adjustments with respect to the central frequency, so small modifications of the square-spiral inductor length were made. In addition, adjustments were made to the distance between resonators. It may seem that the interresonator distance has been changed notably during this first modification for Band-II and Band-III. Actually, the proposed circuit model implies that both the interresonator coupling and the coupling between the feeder and the resonator have been achieved with the first segments of the spiral inductors. The obtained values of $C_{L C}, C_{L L}, C_{C C}, k_{m}$, and $C_{F}$ correspond to this model. This means that the values for couplings will be more accurate for greater spiral lengths, that is, lower frequency bands. For this modification, we decided to implement the interresonator coupling with the third segment of the spiral, for Band-I, and the fourth segment of the spiral, for Band-II and Band-III. This decision is motivated by the fact that the coupling with the third and the fourth segment of the spiral allows us to achieve the same coupling with smaller interresonator distance $[18,24]$. In addition, this modification reduces the footprint. Coupling with the third segment, instead of the fourth one, for Band-I, is used because it provides lower level of parasitic resonance. Frequency responses of the three modified bandpass filters generated by 3D EM and circuit-level simulations are shown in Figures 15(a), 15(c), and 15(e). 3D EM models of the three bandpass filters are shown in Figures 15(b), 15(d), and 15(f). As can be seen, satisfying results have been achieved after only a few modifications. We must emphasize that each of these modifications as well as simulations has taken little time comparing to time necessary to design a filter without knowing the initial dimensions. Comparison of the responses in Figure 15(a) shows discrepancy with respect to the parasitic resonance that occurs at $4.95 \mathrm{GHz}$ for 3D EM model, which is not present for the electric-circuit model. The reason for the appearance of this resonance is spiral inductor length, which behaves as a $\lambda / 4$ transmission line at $1.6 \mathrm{GHz}$. The spiral length is approximately $36 \mathrm{~mm}$ and, at the frequency of $4.95 \mathrm{GHz}$, its electrical length is approximately $3 \pi / 2$. Since the spiral is not grounded at the outer end, the impedance seen at node 3 (Figure 1) is equal to zero at $4.95 \mathrm{GHz}$. This can be validated by replacing the spiral with the $\lambda / 4$ line (at $1.6 \mathrm{GHz}$ ) in the circuit model and observing appearance of this parasitic resonance. Because of its simplicity, our model does not include this phenomenon.

\section{Triple-Bandpass Filter Design}

By incorporating three single bandpass filters into one triple bandpass filter, we obtained the 3D EM model shown in Figure 16(a). The filter dimensions are shown in Figure 16(b). Due to additional interband influences, small adjustments have been made and the final dimensions have been achieved: $d_{1}^{\mathrm{I}}=d_{2}^{\mathrm{II}}=d_{3}^{\mathrm{II}}=0.35 \mathrm{~mm}, d_{1}^{\mathrm{II}}=0.75 \mathrm{~mm}, d_{2}^{\mathrm{I}}=d_{4}^{\mathrm{I}}=d_{4}^{\mathrm{II}}=$ $d_{1}^{\mathrm{III}}=0.5 \mathrm{~mm}, d_{2}^{\mathrm{III}}=0.3 \mathrm{~mm}, d_{3}^{\mathrm{I}}=0.2 \mathrm{~mm}, d_{3}^{\mathrm{III}}=0.4 \mathrm{~mm}$, and $d_{4}^{\mathrm{III}}=0.62 \mathrm{~mm}$.

The block-diagram for the triple-bandpass filter is shown in Figure 17(a). Frequency responses of the triple-bandpass filter, obtained by 3D EM and circuit-level simulations, are shown in Figure 17(b).

It can be noticed that the parasitic resonance (marked with "P" in Figure 17(b)) of Band-I resonators (at $4.95 \mathrm{GHz}$ ) has disappeared. This is due to the vicinity of the two transmission zeros $Z_{2}^{\mathrm{II}}$ and $Z_{1}^{\mathrm{III}}$. Upper transmission zero of Band-II and lower transmission zero of Band-III have suppressed this effect of unwanted resonance. As it was earlier stated, this resonance is determined by the dimensions of the spiral inductor of Band-I. It is possible to adjust spiral dimensions (length and width) to satisfy required central frequency and to simultaneously tune the parasitic resonance to be canceled by the transmission zeros of the other bands.

By comparing the positions of the transmission zeros $Z_{2}^{\mathrm{I}}, Z_{2}^{\mathrm{II}}$, and $Z_{2}^{\mathrm{III}}$ (Figures 15(a), 15(c), and 15(e)) with their positions for the triple-bandpass filter (Figure 17(b)), it is shown that they have been shifted toward the corresponding passbands. This happens for each of the three passbands, when a triple-bandpass filter is realized. The zero shifting occurs because each filter, far enough from its passband, behaves predominantly as a capacitor connected between its 


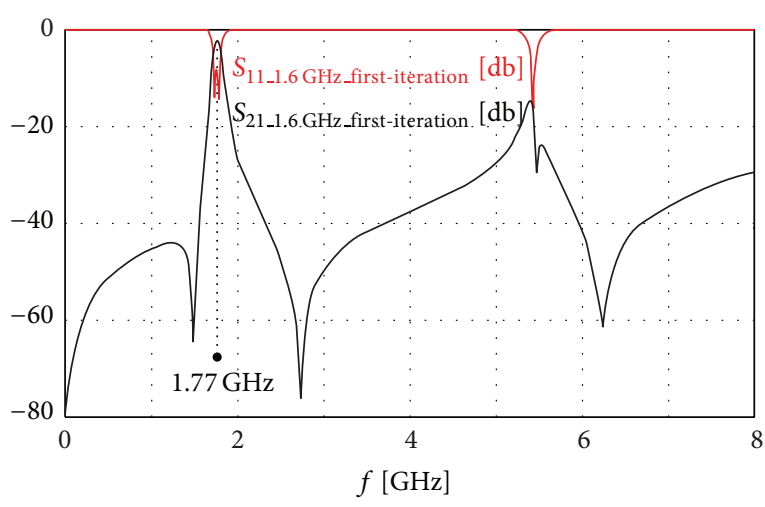

(a)

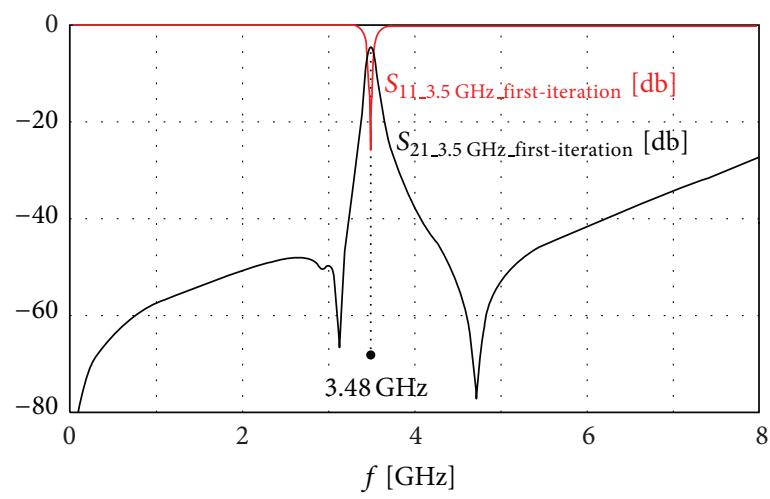

(c)

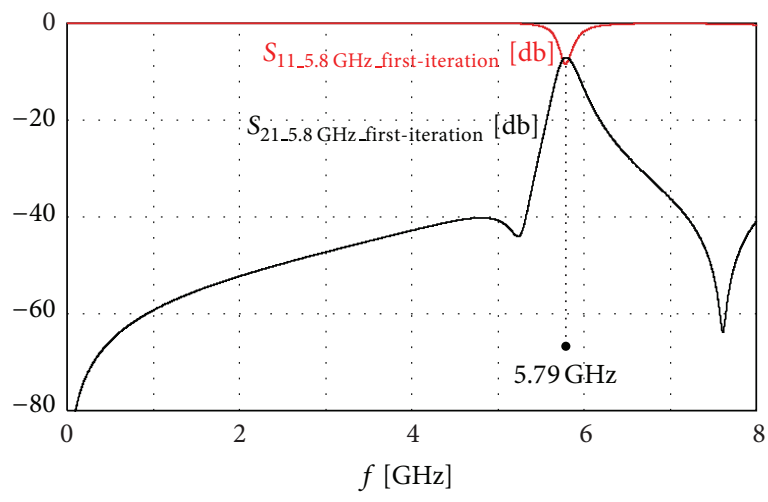

(e)

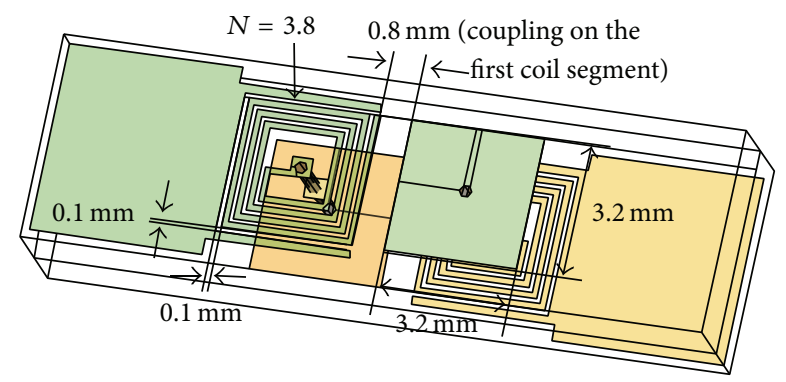

(b)

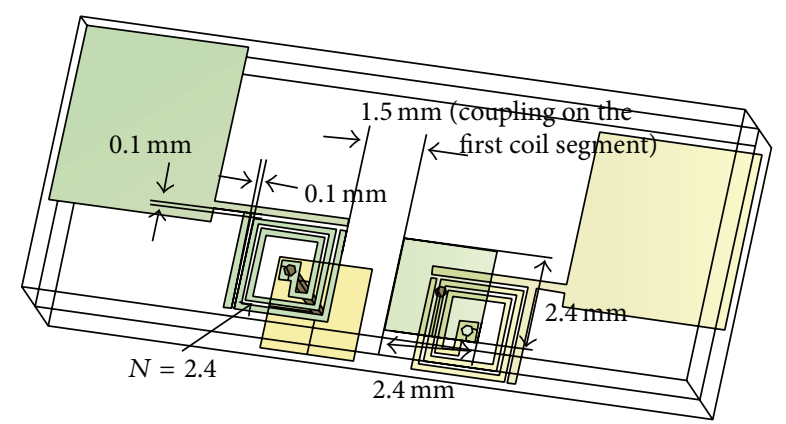

(d)

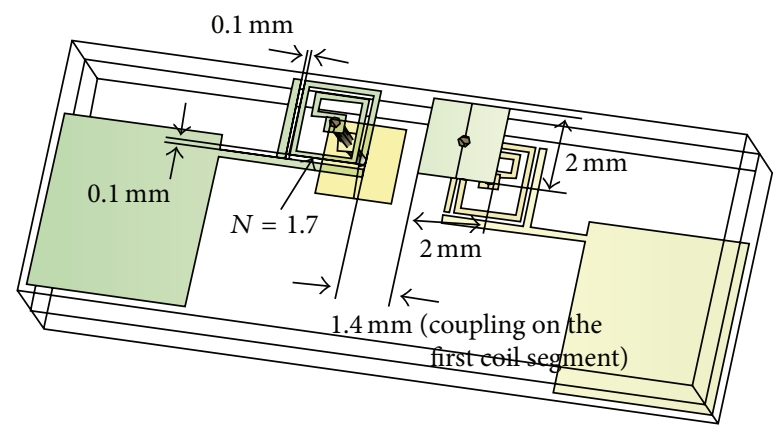

(f)

FIGURE 14: Frequency responses of three bandpass filters generated by 3D EM simulations: (a) Band-I, (c) Band-II, and (e) Band-III. 3D EM model of three bandpass filters: (b) Band-I, (d) Band-II, and (f) Band-III.

input and output ports. This phenomenon is also observed by the circuit-level simulations.

In this final case, the circuit model is used for verification only, not for the design. The values of the circuit lumped elements for the three block-diagrams (in Figure 17(a)) are tuned to match the frequency response obtained by the 3D EM simulation of each filter. This tuning has provided the actual circuit-element values. The spiral inductance and the interspiral capacitance have been tuned to match transmission zeros and retain resonant frequency. The spiral inductances have been changed to $L_{L}^{\mathrm{I}}=28.3 \mathrm{nH}, L_{L}^{\mathrm{II}}=9 \mathrm{nH}$, and $L_{L}^{\mathrm{III}}=4.2 \mathrm{nH}$ (initial values: $L_{L}^{\mathrm{I}}=30.3 \mathrm{nH}, L_{L}^{\mathrm{II}}=$ $11.4 \mathrm{nH}$, and $L_{L}^{\mathrm{III}}=5.2 \mathrm{nH}$ ) and the interspiral capacitance to $C_{\mathrm{IC}}^{\mathrm{I}}=0.11 \mathrm{pF}, C_{\mathrm{IC}}^{\mathrm{II}}=0.1 \mathrm{pF}$, and $C_{\mathrm{IC}}^{\mathrm{III}}=0.07 \mathrm{pF}$ (initial values: $C_{\mathrm{IC}}^{\mathrm{I}}=0.1 \mathrm{pF}, C_{\mathrm{IC}}^{\mathrm{II}}=0.06 \mathrm{pF}$, and $\left.C_{\mathrm{IC}}^{\mathrm{III}}=0.025 \mathrm{pF}\right)$. Additionally, capacitance $C_{\mathrm{IB}}$ (interband capacitance) has been taken into account. This is capacitance between capacitors $C_{C}$ of the first-band and the second-band resonators and also between the second-band and the third-band resonators. Its value is approximately $C_{\mathrm{IB}}=0.05 \mathrm{pF}$. Three individual 


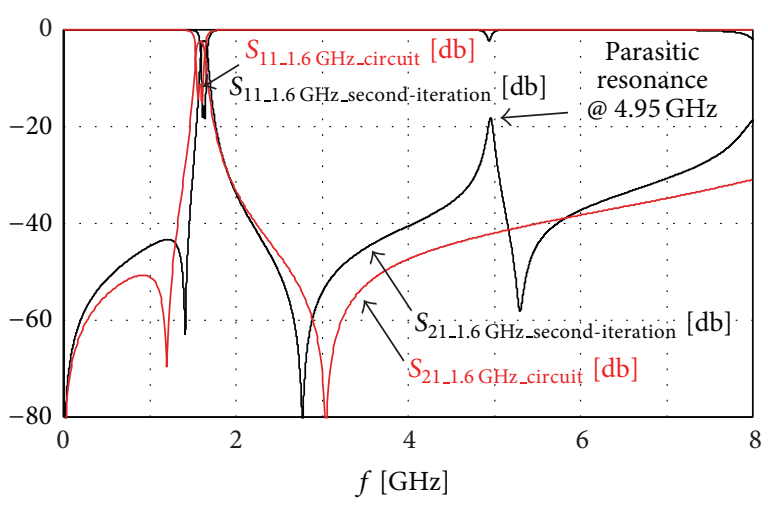

(a)

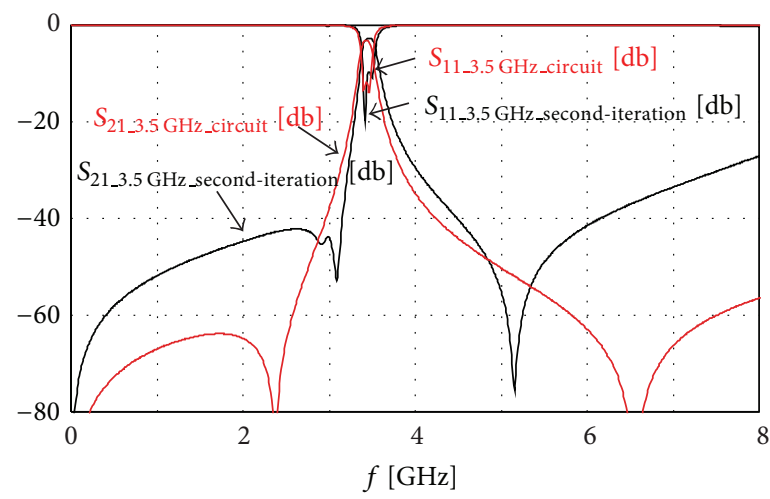

(c)

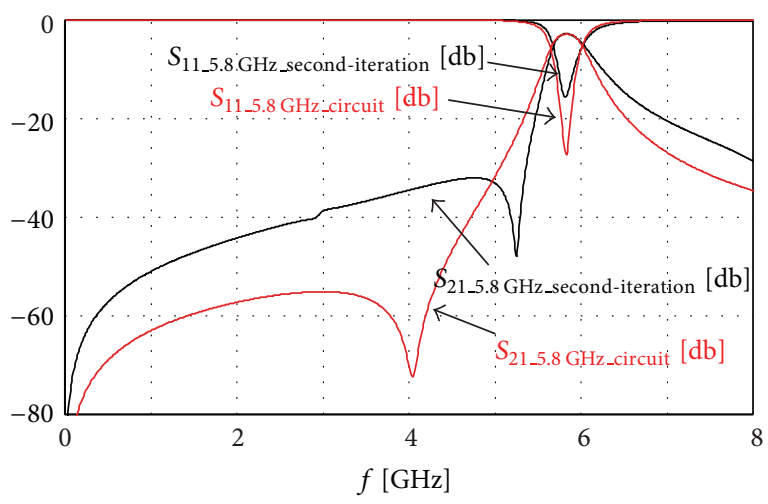

(e)

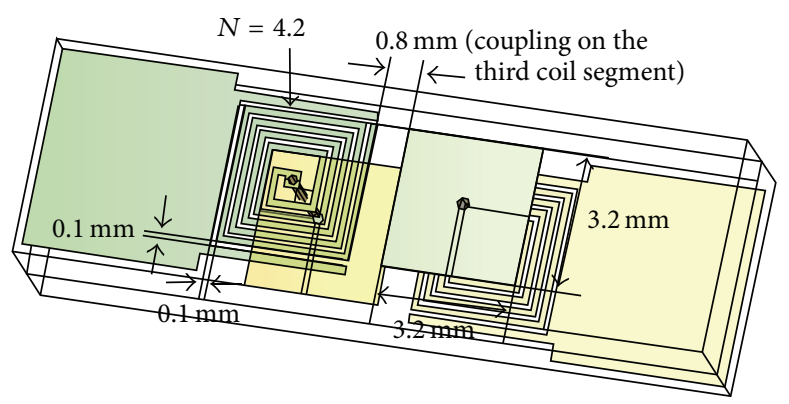

(b)

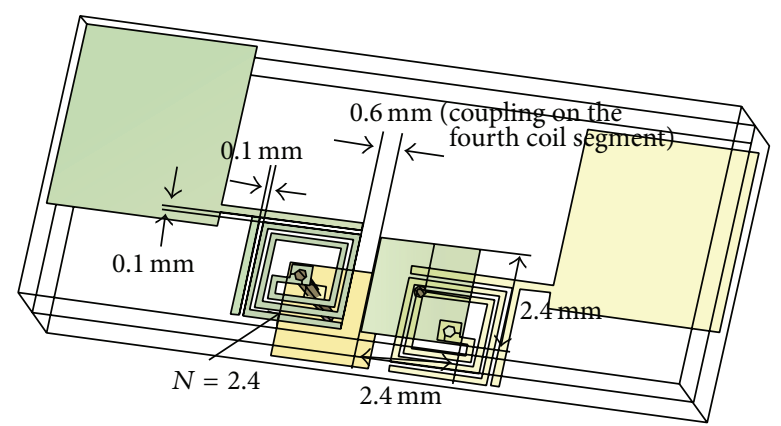

(d)

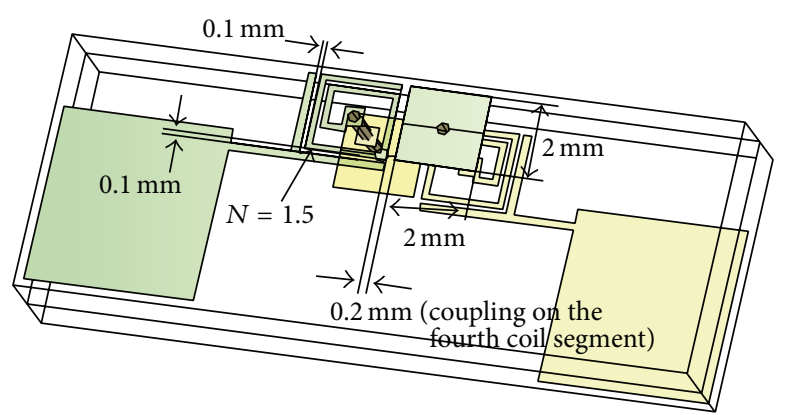

(f)

FIGURE 15: Frequency responses of three bandpass filters generated by 3D EM and circuit-level simulations (after the second adjustment iteration): (a) Band-I, (c) Band-II, and (e) Band-III. 3D EM models of three bandpass filters: (b) Band-I, (d) Band-II, and (f) Band-III.

circuit models comprised into one circuit describe the overall filter characteristic. It should be noted that the circuit-level simulations, using the proposed circuit model, capture all relevant phenomena important for the filter design procedure.

Disagreement between the desired frequency response (obtained by using the circuit models) and the response obtained by 3D EM simulations is greater for higher frequencies, that is, for higher bands. Two reasons explain this behavior. The first one is that our model is based on the frequency independent lumped elements. The second one is that our implementation of feeder-to-resonator coupling and resonator-to-resonator coupling is based on the outer end of the spiral inductor.

\section{Triple-Bandpass Filter Fabrication and Experimental Results}

In the previous section, the procedure for the triple-bandpass filter design has been presented and satisfactory results have been obtained. Following the frequency response of the 3D EM simulations, the triple-bandpass filter is fabricated. The filter is fabricated on RT/Duroid 5880 substrate $\left(\varepsilon_{r}=2.2\right.$, 


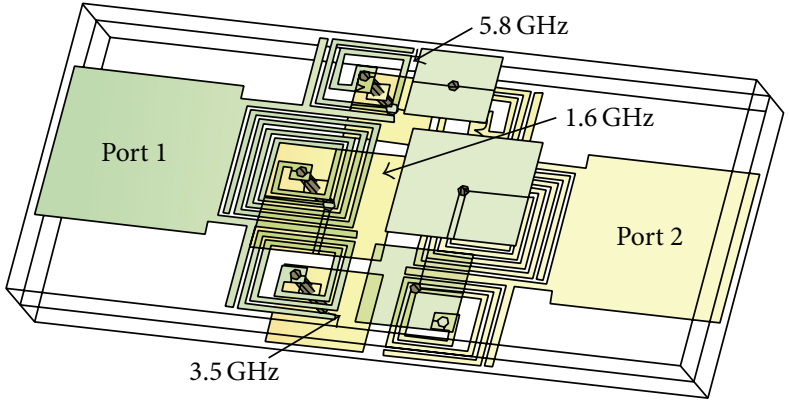

(a)

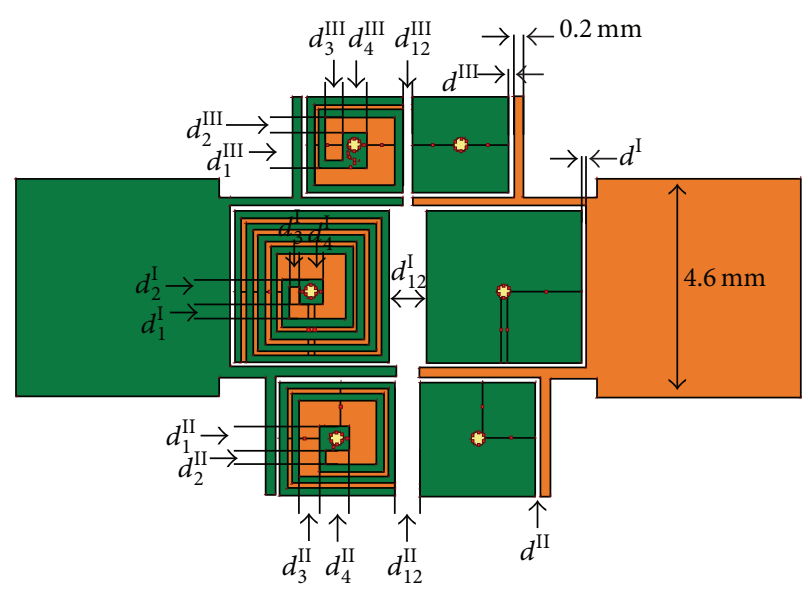

(b)

FIgURE 16: (a) Final 3D EM model of the triple-bandpass filter, (b) dimension details.

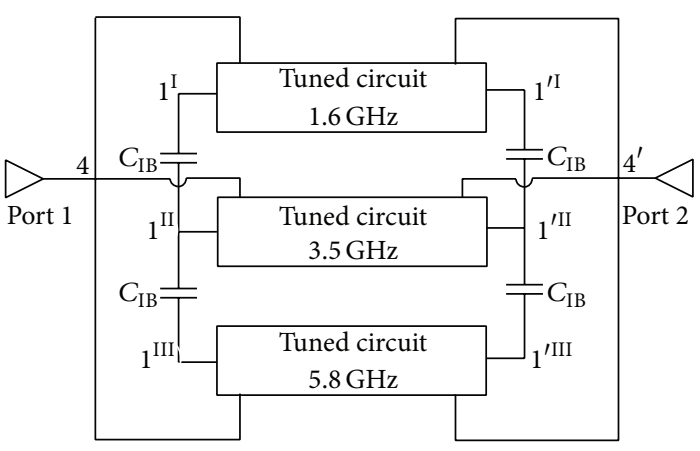

(a)

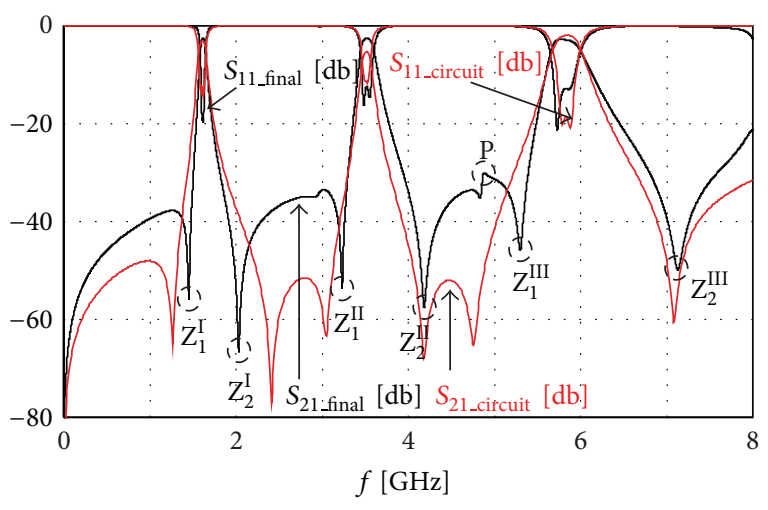

(b)

FIGURE 17: (a) Block diagram of the triple-bandpass filter, (b) frequency responses of the triple-bandpass filter obtained by 3D EM and circuitlevel simulations.
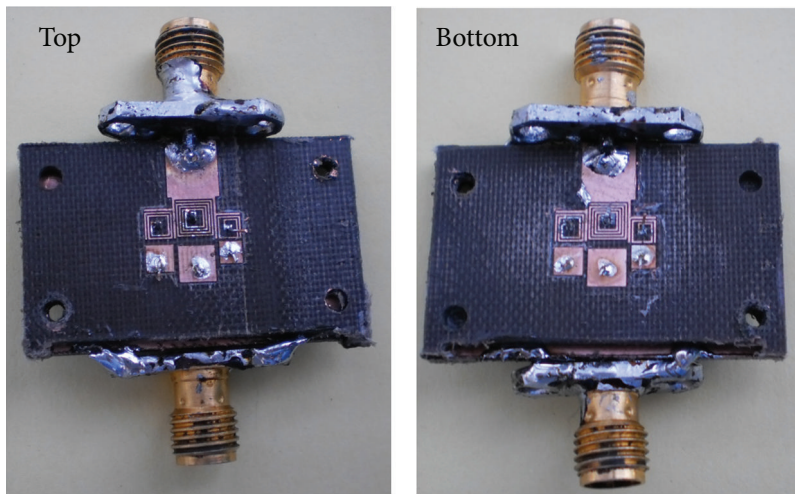

FIGURE 18: Photograph of the fabricated triple-bandpass filter.

$\tan \delta=0.001, h=1.575 \mathrm{~mm}$, and $t=18 \mu \mathrm{m})$. Final filter dimensions (see Figure 16(b)) are as follows (all in millimeters): $d_{12}^{\mathrm{I}}=0.8, d_{12}^{\mathrm{II}}=0.5, d_{12}^{\mathrm{III}}=0.2, d^{\mathrm{I}}=d^{\mathrm{II}}=d^{\mathrm{III}}=0.1$, $d_{3}^{\mathrm{I}}=0.2, d_{1}^{\mathrm{II}}=0.75, d_{2}^{\mathrm{III}}=0.3, d_{3}^{\mathrm{III}}=0.4, d_{4}^{\mathrm{III}}=0.62$,
$d_{2}^{\mathrm{I}}=d_{4}^{\mathrm{I}}=d_{4}^{\mathrm{II}}=d_{1}^{\mathrm{III}}=0.5$, and $d_{1}^{\mathrm{I}}=d_{2}^{\mathrm{II}}=d_{3}^{\mathrm{II}}=0.35$. The photograph of the filter is shown in Figure 18.

The fabricated filter has a footprint of $0.053 \lambda_{g} \times 0.062 \lambda_{g}$, where $\lambda_{g}$ is the guided wavelength of a $50 \Omega$ microstrip line at the central frequency of the first passband (about $7.2 \mathrm{~mm} \times$ $8.4 \mathrm{~mm}$, not including the feed lines).

The proposed size is among the best achieved results (smallest footprint) compared to different design approaches. In single layer techniques, various triple-bandpass filters are summarized in [25], with the best achieved result of $11.2 \mathrm{~mm}$ $\times 9.3 \mathrm{~mm}\left(0.108 \lambda_{g} \times 0.09 \lambda_{g}\right)$ which confirms the benefits in size reduction of the multilayer approach. Our achieved size is better compared to similar approaches with twolayer substrate $31 \mathrm{~mm} \times 29 \mathrm{~mm}\left(0.37 \lambda_{g} \times 0.34 \lambda_{g}\right)$ [26] and $31.4 \mathrm{~mm} \times 29 \mathrm{~mm}\left(0.285 \lambda_{g} \times 0.264 \lambda_{g}\right)$ [27]. Slightly better results are presented in [28] with triple-bandpass filter size of $2.9 \mathrm{~mm} \times 2.4 \mathrm{~mm}\left(0.0527 \lambda_{g} \times 0.0436 \lambda_{g}\right)$ but with the use of an additional substrate and conductive layer, with total of three substrates $\left(\varepsilon_{r}=10.2\right)$ and four conductive layers.

The filter is measured using Agilent N5227A PNA microwave network analyzer. The simulated and measured 


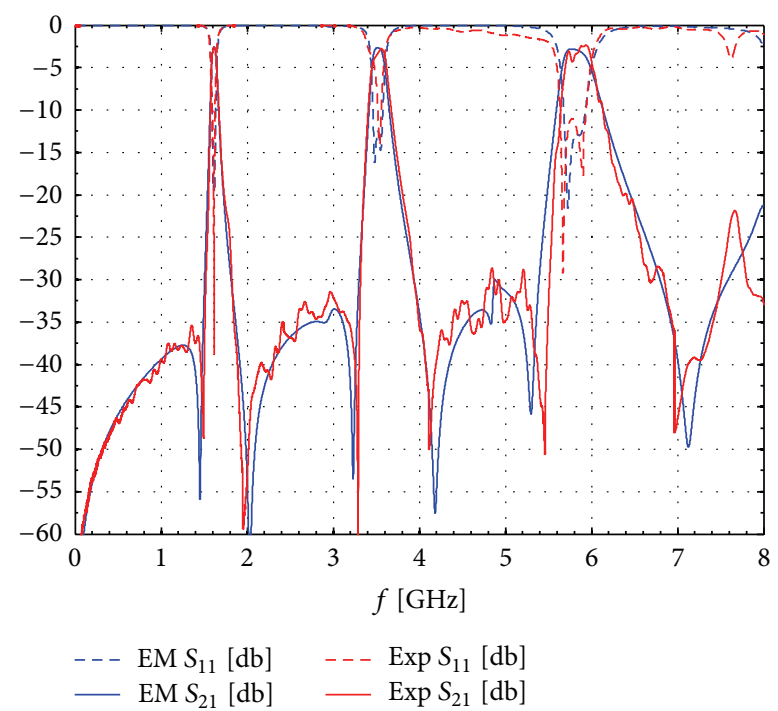

FIGURE 19: Frequency response of the 3D EM simulation and the measured response of the fabricated triple-bandpass filter.

responses of the proposed filter are in good agreement, as shown in Figure 19.

Transmission zeros are located at the frequencies of 1.5 , $1.9,3.3,4.1,5.5$, and $7 \mathrm{GHz}$. Measured $3 \mathrm{~dB}$ bandwidths are $4 \%$, $4.6 \%$, and $7.5 \%$, insertion losses are $2.56,2.68$, and $2.85 \mathrm{~dB}$, and return losses are 15,14 , and $12 \mathrm{~dB}$ at frequencies of 1.6, 3.5, and $5.6 \mathrm{GHz}$, respectively. Unwanted-frequency suppression is greater than $30 \mathrm{~dB}$ between the bands and greater than $20 \mathrm{~dB}$ above the third passband. The most notable difference between the simulated responses and the measured ones is represented by the third passband ripple. This is attributed to the manufacturing process during which vias have been manually soldered to the spiral inductor and patch capacitor. More precise machining process (or tuning the interresonator spacing at the existing model) would certainly lead to the better matching of the obtained results.

\section{Conclusions}

In this paper, we have proposed the use of electric-circuit models for faster design of the multibandpass filters with quasilumped coupled resonators. In addition, we proposed a novel filter which is realized as a multilayer structure. Using the diakoptic approach, the 3D filter structure is decomposed into domains, and each domain is modeled by linear timeinvariant passive electrical elements. The element characteristics have been analytically described, while relations between their electrical and physical parameters have been established.

The fast circuit-level simulators have been used to investigate the influence of each element on the filter characteristic. Two types of interresonator coupling have been investigated, LLCC and LCLC. The limitations of the achievable capacitances, inductances, and mutual inductances have been found. This has yielded the constraints and guidelines in the design of a bandpass filter with the required characteristics.
The values of electric-circuit elements have been mapped to the physical dimensions of the filter elements. This procedure has been used to independently design three bandpass filters which could be combined into one triple-band bandpass filter. Using 3D EM simulations, the obtained triple-band filter structure has been analyzed and modified and the final filter model has been established. The triple-bandpass filter has been fabricated and measured and the results obtained by simulation and measurement are in good agreement. This triple-bandpass filter has also achieved very small size due to the multilayer implementation $(7.2 \mathrm{~mm} \times 8.4 \mathrm{~mm}$, i.e., $\left.0.053 \lambda_{g} \times 0.062 \lambda_{g}\right)$.

During the filter design, the number of time-consuming 3D EM simulations has been reduced significantly, compared to the traditional filter design approach. 3D EM simulations have been used at the last stage of the filter design with the element values obtained from the electric-circuit model. Only a few additional simulations have been needed at the end for fine tuning. Along with the very good results regarding the filter size reduction, we have shown that a significant time saving could be achieved, in the filter analysis and design, by means of modeling the 3D EM structure by an accurate electric circuit.

\section{Conflict of Interests}

The authors declare that there is no conflict of interests regarding the publication of this paper.

\section{Acknowledgment}

This research is supported by the Ministry of Education, Science and Technological Development of the Republic of Serbia under Grant TR32005.

\section{References}

[1] J.-S. Hong, Microstrip Filters for RF/Microwave Applications, John Wiley \& Sons, Hoboken, NJ, USA, 2nd edition, 2011.

[2] M. D. Lutovac, D. V. Tošić, and B. L. Evans, Filter Design for Signal Processing Using MATLAB and Mathematica, Prentice Hall, Upper Saddle River, NJ, USA, 2001, Translated in Chinese, Publishing House of Electronics Industry (PHEI), Beijing, China, 2004.

[3] Q.-X. Chu and H. Wang, "A compact open-loop filter with mixed electric and magnetic coupling," IEEE Transactions on Microwave Theory and Techniques, vol. 56, no. 2, pp. 431-439, 2008.

[4] D. G. Swanson Jr., "Narrow-band microwave filter design," IEEE Microwave Magazine, vol. 8, no. 5, pp. 105-114, 2007.

[5] R. Dhakal and N.-Y. Kim, "A compact symmetric microstrip filter based on a rectangular meandered-line stepped impedance Resonator with a triple-band bandstop response," The Scientific World Journal, vol. 2013, Article ID 457693, 7 pages, 2013.

[6] X. Luo, J.-G. Ma, and E.-P. Li, "Compact high selectivity and wide stopband bandpass filter using dual-layer structure," in Proceedings of the 41st European Microwave Conference (EuMC '11), pp. 460-463, October 2011.

[7] S.-C. Lin, C.-H. Wang, and C. H. Chen, "Novel patch-via-spiral resonators for the development of miniaturized bandpass filters 
with transmission zeros," IEEE Transactions on Microwave Theory and Techniques, vol. 55, no. 1, pp. 137-146, 2007.

[8] J.-S. Hong and M. J. Lancaster, "Aperture-coupled microstrip open-loop resonators and their applications to the design of novel microstrip bandpass filters," IEEE Transactions on Microwave Theory and Techniques, vol. 47, no. 9, pp. 1848-1855, 1999.

[9] C.-H. Chen, C.-H. Huang, T.-S. Horng et al., "Very compact stacked lc resonator-based bandpass filters with a novel approach to tune the transmission zeros," IEEE Microwave and Wireless Components Letters, vol. 19, no. 5, pp. 293-295, 2009.

[10] A. Djaiz and T. A. Denidni, "A new compact microstrip two-layer bandpass filter using aperture-coupled SIR-hairpin resonators with transmission zeros," IEEE Transactions on Microwave Theory and Techniques, vol. 54, no. 5, pp. 1929-1936, 2006.

[11] IE3D-Full-Wave EM Simulation, Optimization, and Synthesis Package, Zeland Software, Inc., Fremont, Calif, USA, 2007.

[12] I. J. Bahl, Lumped Elements for RF and Microwave Circuits, Artech House, Norwood, Mass, USA, 2003.

[13] R. J. Cameron, C. M. Kudsia, and R. R. Mansour, Microwave Filters for Communication Systems: Fundamentals, Design and Applications, John Wiley \& Sons, Hoboken, NJ, USA, 2007.

[14] L.-Y. Ren, "Tri-band bandpass filters based on dual-plane microstrip/DGS slot structure," IEEE Microwave and Wireless Components Letters, vol. 20, no. 8, pp. 429-431, 2010.

[15] Microwave Office, Applied Wave Research, El Segundo, Calif, USA, 2009, http://www.appwave.com/.

[16] M. M. Potrebić and D. V. Tošić, "A novel design of a compact multilayer resonator using double-sided microstrip," Optoelectronics and Advanced Materials: Rapid Communications, vol. 6, no. 3-4, pp. 441-445, 2012.

[17] D. Miljanović, M. Potrebić, D. V. Tošić, and Z. Stamenković, "Feeder realization for quasi-lumped multilayer resonators with low Q-factor," in Proceedings of the 11th WSEAS International Conerence on Circuits, Systems, Electronics, Control \& Signal Processing (CSECS '12), pp. 63-68, December 2012.

[18] D. M. Miljanović, M. M. Potrebić, D. V. Tošić, and Z. Stamenković, "Design of miniaturized bandpass filters using quasilumped multilayer resonators," Journal of Circuits, Systems and Computers, vol. 23, no. 6, Article ID 1450083, 21 pages, 2014.

[19] M. Hosseini, G. Zhu, and Y.-A. Peter, "A new formulation of fringing capacitance and its application to the control of parallel-plate electrostatic micro actuators," Analog Integrated Circuits and Signal Processing, vol. 53, no. 2-3, pp. 119-128, 2007.

[20] M. E. Golfarb and R. A. Pucel, "Modeling via hole grounds in microstrip," IEEE Microwave and Guided Wave Letters, vol. 1, no. 6, pp. 135-137, 1991.

[21] R. Melati, A. Hamid, L. Thierry, and M. Derkaoui, "Design of a new electrical model of a ferromagnetic planar inductor for its integration in a micro-converter," Mathematical and Computer Modelling, vol. 57, no. 1-2, pp. 200-227, 2013.

[22] Mathematica, Wolfram Research, Inc., Champaign, Ill, USA, 2013, http://www.wolfram.com.

[23] MITS Electronics, PCB Prototyping Machine MITS FP-21TP Precision, MITS Electronics, Tokyo, Japan, 2008, http://www .mitspcb.com

[24] J.-S. Hong and M. J. Lancaster, "Couplings of microstrip square open-loop resonators for cross-coupled planar microwave filters," IEEE Transactions on Microwave Theory and Techniques, vol. 44, no. 11, pp. 2099-2109, 1996.
[25] H.-W. Liu, Y. Wang, X.-M. Wang et al., "Compact and high selectivity tri-band bandpass filter using multimode steppedimpedance resonator," IEEE Microwave and Wireless Components Letters, vol. 23, no. 10, pp. 536-538, 2013.

[26] Y.-F. Chen, S.-H. Huang, H.-W. Wu, and H.-Y. Lee, "Design of compact tri-band bandpass filter using multilayer substrate technique," in Proceedings of the Asia-Pacific Microwave Conference (APMC '13), pp. 527-529, Seoul, Republic of Korea, November 2013.

[27] P.-Y. Hsiao and R.-M. Weng, "A tri-band bandpass filter using 2T4L2C-pattern multi-layer structure," in Proceedings of the Asia Pacific Microwave Conference (APMC '09), pp. 1416-1418, Singapore, December 2009.

[28] C.-H. Chen, C.-H. Huang, T.-S. Horng, and S.-M. Wu, "Highly miniaturized multiband bandpass filter design based on a stacked spiral resonator structure," IEEE Transactions on Microwave Theory and Techniques, vol. 60, no. 5, pp. 1278-1286, 2012. 


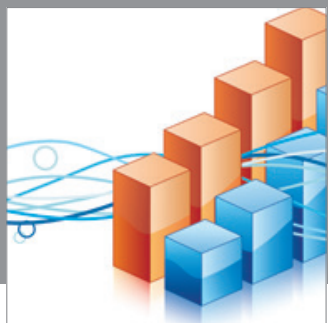

Advances in

Operations Research

mansans

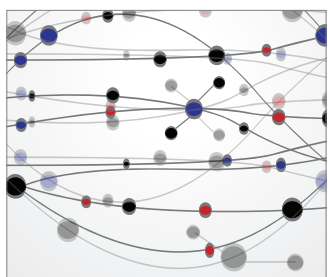

The Scientific World Journal
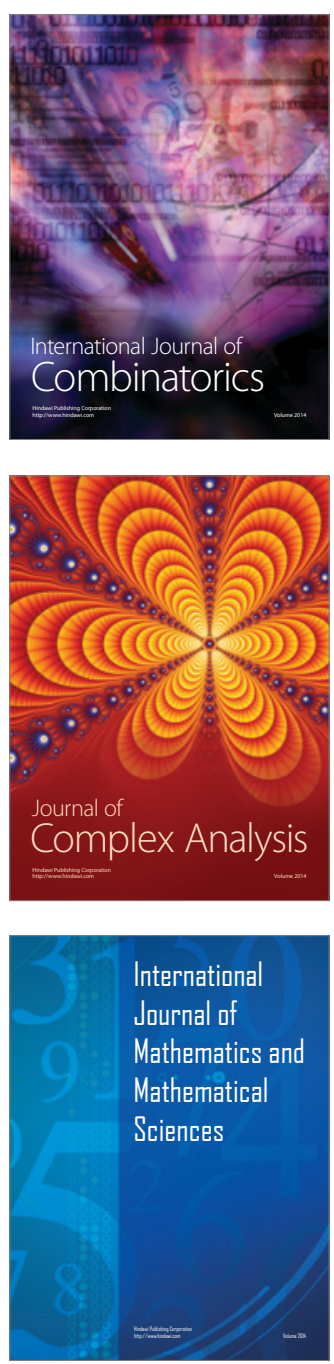
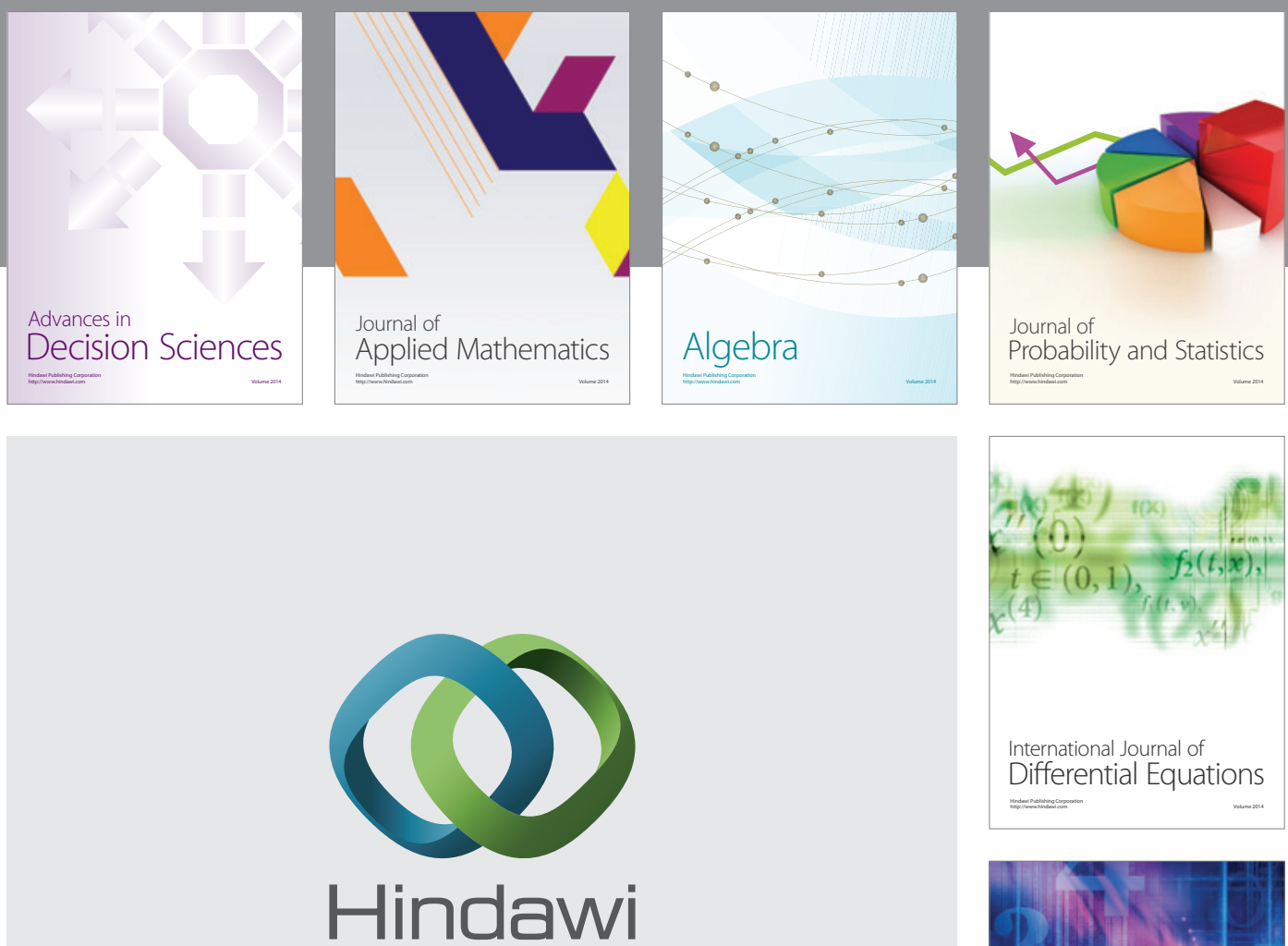

Submit your manuscripts at http://www.hindawi.com
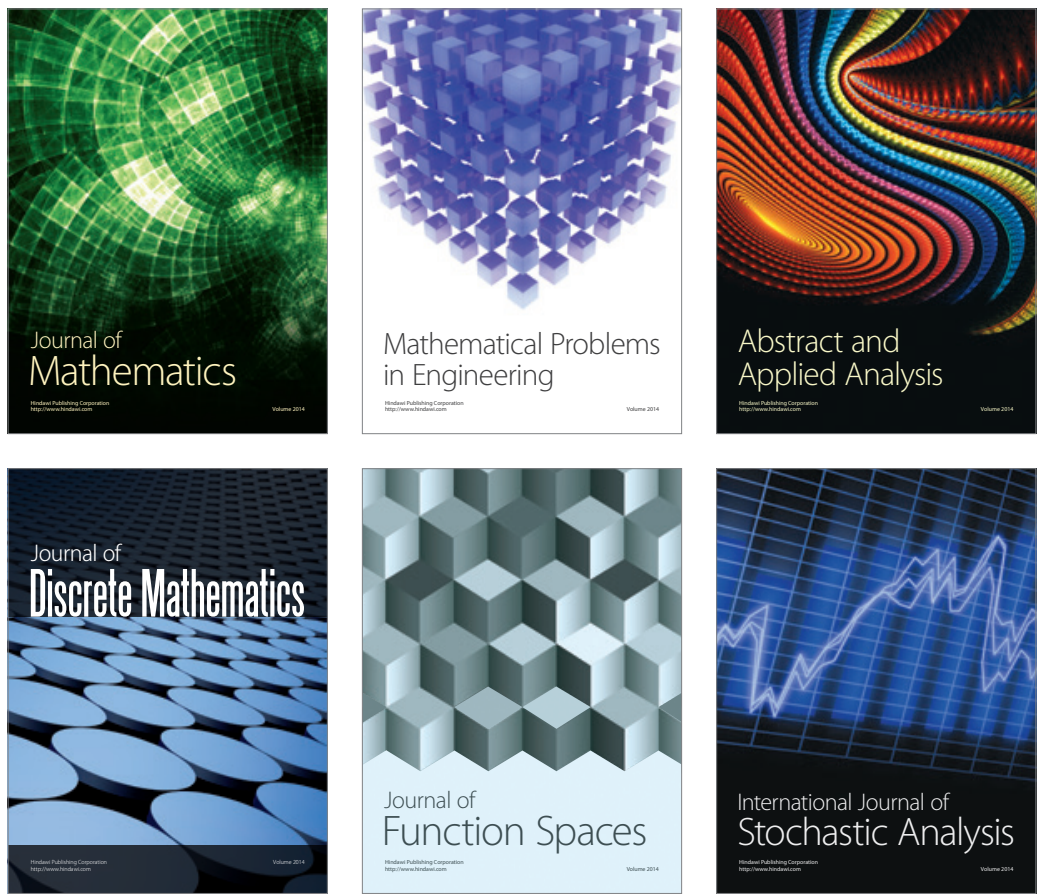

Journal of

Function Spaces

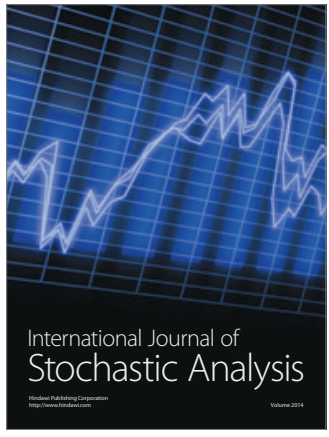

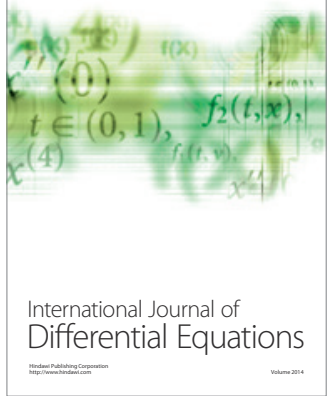
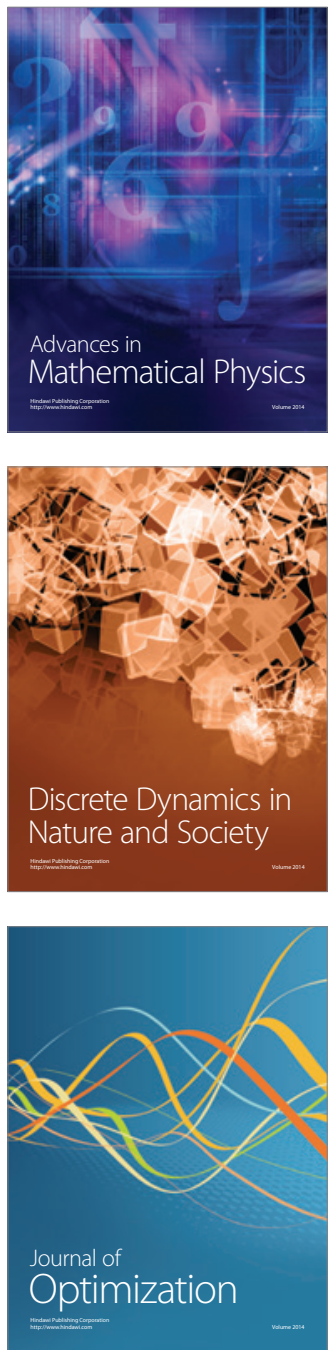